Louisiana State University

LSU Digital Commons

4-1-2017

\title{
Extensions of Razumikhin's theorem and Lyapunov-Krasovskii functional constructions for time-varying systems with delay
}

Frédéric Mazenc

Laboratoire des Signaux et Systèmes

Michael Malisoff

Louisiana State University

Follow this and additional works at: https://digitalcommons.Isu.edu/mathematics_pubs

\section{Recommended Citation}

Mazenc, F., \& Malisoff, M. (2017). Extensions of Razumikhin's theorem and Lyapunov-Krasovskii functional constructions for time-varying systems with delay. Automatica, 78, 1-13. https://doi.org/ 10.1016/j.automatica.2016.12.005

This Article is brought to you for free and open access by the Department of Mathematics at LSU Digital Commons. It has been accepted for inclusion in Faculty Publications by an authorized administrator of LSU Digital Commons. For more information, please contact ir@lsu.edu. 


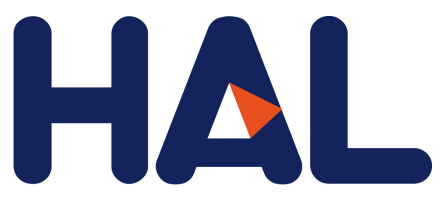

archives-ouvertes

\section{Extensions of Razumikhin's Theorem and Lyapunov-Krasovskii Functional Constructions for Time-Varying Systems with Delay*}

Frédéric Mazenc, Michael Malisoff

\section{To cite this version:}

Frédéric Mazenc, Michael Malisoff. Extensions of Razumikhin's Theorem and Lyapunov-Krasovskii Functional Constructions for Time-Varying Systems with Delay *. Automatica, Elsevier, 2017, 78, pp.1-13. 10.1016/j.automatica.2016.12.005 . hal-01660097

\section{HAL Id: hal-01660097 https://hal.inria.fr/hal-01660097}

Submitted on 9 Dec 2017

HAL is a multi-disciplinary open access archive for the deposit and dissemination of scientific research documents, whether they are published or not. The documents may come from teaching and research institutions in France or abroad, or from public or private research centers.
L'archive ouverte pluridisciplinaire HAL, est destinée au dépôt et à la diffusion de documents scientifiques de niveau recherche, publiés ou non, émanant des établissements d'enseignement et de recherche français ou étrangers, des laboratoires publics ou privés. 


\title{
Extensions of Razumikhin's Theorem and Lyapunov- Krasovskii Functional Constructions for Time-Varying Systems with Delay*
}

\author{
Frédéric Mazenc $^{\mathrm{a}}$ and Michael Malisoff ${ }^{\mathrm{b}}$ \\ ${ }^{a}$ EPI DISCO Inria-Saclay, Laboratoire des Signaux et Systèmes, CNRS, CentraleSupélec, Université Paris-Sud, \\ 3 rue Joliot Curie, 91192, Gif-sur-Yvette, France. \\ ${ }^{\mathrm{b}}$ Department of Mathematics, Louisiana State University, Baton Rouge, LA 70803-4918, USA.
}

\begin{abstract}
We prove extensions of Razumikhin's theorem for time-varying continuous and discrete time nonlinear systems. Our results include a novel 'strictification' technique for converting a nonstrict Lyapunov function into a strict one. We also provide new constructions of Lyapunov-Krasovskii functionals that can be used to prove robustness to perturbations. Our examples include a key model from identification theory, and they show how our method can sometimes allow broader classes of delays than the results in the literature.
\end{abstract}

Key words: Delay, robustness, stability, time-varying

\section{Introduction}

Input delays are ubiquitous in engineering, where they can model time consuming information gathering or latencies; see Bekiaris-Liberis and Krstic (2013a), Bekiaris-Liberis and Krstic (2013b), Dieulot and Richard (2001), Downey et al. (2016), Gu et al. (2003), Gu and Niculescu (2003), Marquez et al. (2015), Mazenc and Niculescu (2011), Mondié and Michiels (2003), and Petit et al. (1998). However, such systems are usually too complicated to be covered by standard methods for undelayed systems; see Richard (2003). Hence, this note builds on our research (begun in Mazenc and Malisoff (2015b), Mazenc et al. (2014), and Mazenc et al. (2015)) on novel methods to prove important stability properties for time delayed systems.

Since the flow map for a nonlinear system usually cannot be written in explicit closed form, it is natural to use indirect Lyapunov approaches to prove stability for undelayed systems. Lyapunov functions provide a generalized notion of energy in dynamical systems, so the decay of a Lyapunov function often implies asymptotic convergence of solutions towards an equilibrium. Classical Lya-

\footnotetext{
* Corresponding author: F. Mazenc. A preliminary version appeared in the proceedings of the 2016 American Control Conference; see the end of Section 1 for the differences between the conference version and this paper. Malisoff was supported by NSF-ECCS Grants 1102348 and 1408295.

Email addresses:

frederic.mazenc@12s. centralesupelec.fr (Frédéric

Mazenc), malisoff@lsu.edu (Michael Malisoff).
}

punov function approaches require that the time derivative of the Lyapunov function be nonpositive along all solutions, which can sometimes be a demanding condition, especially for time-varying or time delay systems. While classical Lyapunov functions are suited for proving stability of systems without delays, one often replaces Lyapunov functions by Lyapunov-Krasovskii or Razumikhin functions to help solve stability problems for delayed systems; see Fridman and Niculescu (2008). In particular, if we consider the dynamics for the extended state $q(t)=\left(q_{1}(t), q_{2}(t)\right)=$ $(x(t), x(t-\tau))$ of a system $\dot{x}(t)=f(t, x(t), x(t-\tau))$ with a constant delay $\tau$, then we obtain $\dot{q}_{2}(t)=f(t-\tau, q(t-\tau))$, so we cannot eliminate the delay from the system by simply considering the dynamics for the extended state $q(t)$.

As noted in Mazenc et al. (2014) and Zhou (2014), timevarying systems with delay are important, e.g., for tracking problems. The works Bresch-Pietri and Petit (2014), Mazenc and Malisoff (2015a), and Mazenc et al. (2015) are significant, in part because they use Lyapunov functionals to prove stability but allow the time derivatives of the functionals to take positive values along trajectories. For delayed systems, one often builds Lyapunov-Krasovskii functionals by adding together (a) a Lyapunov function for the corresponding undelayed system and (b) an integral involving the delay, whose integrand is a function of the state; see Mazenc et al. (2008). Applying Razumikhin's theorem does not generally involve such integral terms; see Gu et al. (2003), Hale and Verduyn-Lunel (1993), and (Zhou, 2014, Theorem B.2). Razumikhin's approach is useful under time-varying delays. Our work Mazenc and Mal- 
isoff $(2015 a)$ pursues a different approach, involving neither Krasovskii nor Razumikhin functionals.

This paper extends Razumikhin's theorem for time-varying systems. We extend the strictification technique, developed in Malisoff and Mazenc (2009). Our first result does not use periodicity. However, due to the importance of periodic systems, we later present a simpler result in the periodic case. We obtain less conservative stability conditions than those in Mazenc et al. (2015). We also provide new constructions for input-to-state stable (or ISS) LyapunovKrasovskii functionals for continuous time delay systems; see, e.g., , Pepe and Jiang (2006) and Pepe et al. (2008) for ISS Lyapunov-Krasovskii functionals and analogs for discrete time systems with delay, which are important for discretization and sampling (as explained, e.g., in Astrom and Wittenmark (1996), Karafyllis and Krstic (2012) and Montagner et al. (2005)). See, e.g., Cloosterman et al. (2007) for motivation for discrete time systems, using networked systems that are inspired by communication networks.

Our four examples below demonstrate the value of our theory. In Section 6.5, we illustrate our findings in a model from identification theory with a time-varying delay, building on our treatment of this dynamics in Mazenc et al. (2008) where the delays were constant. The preliminary version of this paper is Mazenc and Malisoff (2016), which did not include our ISS Lyapunov-Krasovskii functional constructions, nor did it cover discrete time systems. Also, the present paper includes three illustrations that were not in Mazenc and Malisoff (2016).

\section{Definitions and Notation}

Throughout this work, all dimensions are arbitrary, unless indicated otherwise. The usual Euclidean norm, and its induced matrix norm, are denoted by $|\cdot|$, and $\left.|\cdot|\right|_{\mathcal{I}}$ denotes the (essential) supremum over any interval $\mathcal{I} \subseteq \mathbb{R}$. Let $C^{1}$ be the set of all continuously differentiable functions, whose domains and ranges will be clear from the context. For each constant delay bound $\tau$, let $C\left([-\tau, 0], \mathbb{R}^{n}\right)$ be the set of all continuous $\mathbb{R}^{n}$-valued functions that are defined on $[-\tau, 0]$, which we denote by $C_{\text {in }}$ and call the set of all initial functions. For each continuous function $\varphi:[-\tau, \infty) \rightarrow \mathbb{R}^{n}$ and $t \geq 0$, set $\varphi_{t}(m)=\varphi(t+m)$ for all $m \in[-\tau, 0]$. A locally bounded function $\phi$ defined on an interval $\mathcal{I} \subseteq \mathbb{R}$ is called piecewise continuous provided that for each bounded set $S \subseteq \mathcal{I}$, the restriction of $\phi$ to $S$ has only finitely many points where it is discontinuous (which includes continuous functions as a special case). When we say that a function $\phi$ defined on $[0, \infty) \times \mathbb{R}^{n}$ is differentiable on $\left([0, \infty) \times \mathbb{R}^{n}\right) \backslash\{0\}$, we view its partial derivative $\phi_{t}(0, x)$ with respect to its first argument as a right derivative at 0 for each $x \neq 0$.

Let $\mathcal{K}$ denote the set of all strictly increasing continuous functions $\alpha:[0, \infty) \rightarrow[0, \infty)$ such that $\alpha(0)=0$, and $\mathcal{K}_{\infty}$ denote the set of all unbounded class $\mathcal{K}$ functions. A function $F:[0, \infty) \times C_{\text {in }} \rightarrow \mathbb{R}^{n}$ is called uniformly bounded with respect to its first argument provided that there are a function $\alpha \in \mathcal{K}_{\infty}$ and a constant $\bar{c}>0$ such that
$|F(t, \phi)| \leq \bar{c}+\alpha\left(|\phi|_{[-\tau, 0]}\right)$ holds for all $t \geq 0$ and $\phi \in C_{\text {in }}$; it is called Lipschitz continuous with respect to its second argument provided that there is a function $\alpha \in \mathcal{K}_{\infty}$ such that for each constant $\bar{K}>0$, we have

$$
|F(t, \phi)-F(t, \psi)| \leq \alpha(\bar{K})|\phi-\psi|_{[-\tau, 0]}
$$

for all $t \geq 0$ and for all $\phi$ and $\psi$ in $C_{\text {in }}$ such that $\max \left\{|\phi|_{[-\tau, 0]},|\psi|_{[-\tau, 0]}\right\} \leq \bar{K}$. Also, we use the standard definitions of input-to-stability (or ISS, which we also use to abbreviate input-to-state stable) and ISS LyapunovKrasovskii functionals; see Zhou (2014) for their standard formulations for delay systems. Finally, for each $s \in \mathbb{R}$, we let Floor $(s)$ denote the largest integer $J$ such that $J \leq s$.

\section{General Result for Continuous Time Unper- turbed Systems}

\subsection{Statement of Result}

We consider a nonlinear time-varying system

$$
\dot{x}=F\left(t, x_{t}\right)
$$

whose (possibly time-varying) delay is bounded by some constant $\tau$, having initial conditions in $C_{\text {in }}$ (but see below for analogs for systems with perturbations). While stated for systems with state delays, our results apply to systems with input delays as well, by viewing (2) as a closed loop system, with $F\left(t, x_{t}\right)=G\left(t, x_{t}, u\left(x_{t}\right)\right)$ for an open loop dynamics $\dot{x}(t)=G\left(t, x_{t}, u\right)$ and a control $u\left(x_{t}\right)$. We assume:

Assumption 1 The function $F$ is uniformly bounded with respect to its first argument and Lipschitz continuous with respect to its second argument. Also, there exist a function $V:[0, \infty) \times \mathbb{R}^{n} \rightarrow[0, \infty)$ and functions $\alpha_{1}$ and $\alpha_{2}$ of class $\mathcal{K}_{\infty}$ such that

$$
\alpha_{1}(|x|) \leq V(t, x) \leq \alpha_{2}(|x|)
$$

hold for all $(t, x) \in[0, \infty) \times \mathbb{R}^{n}$ and such that there are bounded piecewise continuous functions $a: \mathbb{R} \rightarrow \mathbb{R}$ and $b: \mathbb{R} \rightarrow[0, \infty)$ such that

$$
\frac{d}{d t} V(t, x(t)) \leq a(t) V(t, x(t))+b(t) \sup _{\ell \in[t-\tau, t]} V(\ell, x(\ell))
$$

holds along all trajectories of (2).

Assumption 2 There are a positive constant $\beta$ and $a$ bounded piecewise continuous function $\epsilon: \mathbb{R} \rightarrow \mathbb{R}$ such that

$$
\mu(t)=a(t)+b(t)
$$

with the choices of a and $b$ from Assumption 1, satisfies

$$
\left|\int_{0}^{t}(\epsilon(\ell)+\mu(\ell)) \mathrm{d} \ell\right| \leq \beta
$$

for all $t \geq 0$. Moreover, the function

$$
\kappa(t)=\sup _{\ell \in[t-\tau, t]} \int_{\ell}^{t}(-\epsilon(s)-\mu(s)) \mathrm{d} s
$$

admits a constant $\varpi>0$ such that

$$
\sup _{t \geq 0}\left[\left(e^{\kappa(t)}-1\right) b(t)-\epsilon(t)\right] \leq-\varpi
$$

holds. 
See below for interesting applications where we can easily check the preceding assumptions. We prove:

Theorem 1 If Assumptions 1-2 hold, then (2) is globally uniformly asymptotically stable at the origin.

Remark 1 To better understand the significance of the preceding theorem, consider the special case where there is a constant $\underline{\mu}>0$ such that the function (5) satisfies

$$
\mu(t) \leq-\underline{\mu} \text { for all } t \geq 0,
$$

and let $\bar{b}>0$ be any constant bound for $b$. Choose any constant $q \in(1,1+\underline{\mu} /(2 \bar{b}))$. Then, for all $t \geq 0$,

$$
a(t)+q b(t)=\mu(t)+(q-1) b(t) \leq-\underline{\mu} / 2 .
$$

Hence, if $q V(t, x(t)) \geq \sup _{\ell \in[t-\tau, t]} V(\ell, x(\ell))$ for some $t \geq$ 0 , then the nonnegativity of $V$ and $b$ and (4) imply that

$$
\begin{aligned}
\frac{d}{d t}(V(t, x(t))) & \leq a(t) V(t, x(t))+b(t) q V(t, x(t)) \\
& \leq-(\underline{\mu} / 2) V(t, x(t))
\end{aligned}
$$

are satisfied. Then Razumikhin's theorem ensures the global uniform asymptotic stability of the origin of (2). However, our objective is precisely to establish stability results in cases where (9) may not be satisfied. Our assumptions allow the function a to take positive and negative values. See Section 6 for an analysis in the special case of periodic systems, which further explains the motivation for Assumption 2. While our decay estimate (4) is reminiscent of (A.1) in (Mazenc et al., 2015, Lemma A.1), a valuable feature of Theorem 1 is that it does not require the contractiveness assumption that was made in (Mazenc et al., 2015, Lemma A.1).

\subsection{Proof of Theorem 1}

Throughout the sequel, all inequalities and equalities are for all $t \geq 0$ and along all solutions of (2), unless otherwise indicated. Assumption 1 ensures the standard existence and uniqueness properties for solutions of (2). Let $\bar{a}>0$, $\bar{b}>0$, and $\bar{\epsilon}>0$ be any constant bounds for $|a(t)|, b(t)$, and $|\epsilon(t)|$, respectively. We define

$$
\begin{aligned}
& \theta(t)=\exp \left(-\int_{0}^{t}(\epsilon(s)+\mu(s)) \mathrm{d} s\right) \text { and } \\
& U(t, x)=\theta(t) V(t, x) .
\end{aligned}
$$

Then for all $\ell \in[t-\tau, t]$, we have

$$
\frac{\theta(t)}{\theta(\ell)} \leq e^{\kappa(t)} .
$$

Also, (4)-(5) give

$$
\begin{aligned}
\frac{d}{d t}(U(t, x(t)))= & \dot{\theta}(t) V(t, x(t))+\theta(t) \frac{d}{d t}(V(t, x(t))) \\
\leq & -(\epsilon(t)+\mu(t)) U(t, x(t)) \\
& +a(t) U(t, x(t)) \\
& +\theta(t) b(t) \sup _{\ell \in[t-\tau, t]} V(\ell, x(\ell)) \\
= & (-\epsilon(t)-b(t)) U(t, x(t)) \\
& +b(t) \sup _{\ell \in[t-\tau, t]} \theta(t) V(\ell, x(\ell)) .
\end{aligned}
$$

It follows from (13) that

$$
\begin{aligned}
\frac{d}{d t}(U(t, x(t))) \leq & -(\epsilon(t)+b(t)) U(t, x(t)) \\
& +b(t) \sup _{\ell \in[t-\tau, t]} \frac{\theta(t)}{\theta(\ell)} \\
& \times \sup _{\ell \in[t-\tau, t]} U(\ell, x(\ell)) \\
\leq & -(\epsilon(t)+b(t)) U(t, x(t)) \\
& +e^{\kappa(t)} b(t) \sup _{\ell \in[t-\tau, t]} U(\ell, x(\ell)) .
\end{aligned}
$$

Also, we can use the nonnegativity of $b$ to get

$$
\begin{aligned}
e^{\kappa(t)} b(t) & \leq \exp \left(\int_{t-\tau}^{t}|\epsilon(s)+\mu(s)| \mathrm{d} s\right) b(t) \\
& \leq e^{\tau(\bar{\epsilon}+\bar{a}+\bar{b})} \bar{b} .
\end{aligned}
$$

On the other hand, for all $r>0$ such that $r e^{\tau(\bar{\epsilon}+\bar{a}+\bar{b})} \bar{b} \leq \frac{\varpi}{2}$, we can use (15) to check that

$$
r e^{\kappa(t)} b(t) \leq \frac{\varpi}{2}
$$

Also, (8) ensures that for all $t \geq 0$, we have

$$
\left(e^{\kappa(t)}-1\right) b(t)-\epsilon(t) \leq-\varpi
$$

From (16) and (17), it follows that for all $t \geq 0$, we have

$$
\left(e^{\kappa(t)}-1\right) b(t)+r e^{\kappa(t)} b(t)-\epsilon(t) \leq-\frac{\varpi}{2} .
$$

Set $q=1+r$. Then grouping terms gives $\left(q e^{\kappa(t)}-1\right) b(t)-$ $\epsilon(t) \leq-\frac{\varpi}{2}$.

Next note that when

$$
q U(t, x(t)) \geq \sup _{\ell \in[t-\tau, t]} U(\ell, x(\ell)),
$$

the second inequality in (14) gives

$$
\begin{aligned}
\frac{d}{d t}(U(t, x(t)) \leq & -(\epsilon(t)+b(t)) U(t, x(t)) \\
& +e^{\kappa(t)} b(t) q U(t, x(t)) \\
\leq & -\frac{\varpi}{2} U(t, x(t)) .
\end{aligned}
$$

Also, (6) and Assumption 1 give

$$
e^{-\beta} \alpha_{1}(|x|) \leq U(t, x) \leq e^{\beta} \alpha_{2}(|x|)
$$

The theorem now follows from the classical Razumikhin theorem, by combining (19)-(20).

\section{ISS Lyapunov-Krasovskii Functionals}

Before turning to our discrete time results, we present new constructions for ISS Lyapunov-Krasovskii functionals, which are useful tools for the stability analysis of nonlinear systems with delays or for the design of control laws; see the examples section below. In particular, ISS Lyapunov-Krasovskii functionals make it possible to prove that a system possesses the ISS robustness property, and are useful when one aims to establish local asymptotic results or find estimates of basins of attraction. For simplicity, the strictification results in this section assume that we have time-varying systems with constant pointwise and distributed delays, but we can also cover time-varying delays 
as discussed in the preceding sections. It is important to study systems with distributed delays because frequently systems with pointwise delays are transformed into systems with distributed delays to ease the analysis. Throughout this section, we study nonlinear time-varying systems

$$
\dot{x}=F\left(t, x_{t}, \xi(t)\right)
$$

with delays and initial conditions in $C_{\text {in }}$, where $\tau \geq 0$ is an upper bound on the delays, $\xi:[0, \infty) \rightarrow \mathbb{R}^{p}$ is a piecewise continuous disturbance, and $F$ is a continuous function that is locally Lipschitz with respect to its $x_{t}$ argument. In addition, we assume throughout this section that the following holds:

Assumption 3 There is a function $K$ of class $\mathcal{K}$ such that

$$
|F(t, \phi, \xi)| \leq K\left(|\phi|_{\infty}+|\xi|\right)
$$

holds for all $t \geq 0, \phi \in C_{\mathrm{in}}$, and $\xi \in \mathbb{R}^{p}$.

In Section 4.1, we provide a general Lyapunov-Krasovskii functional construction that uses a suitable nonstrict Lyapunov-like function. Then in Section 4.2, we provide an alternative construction that leads to a large class of systems for which the assumptions from Section 4.1 hold.

\subsection{Construction of Lyapunov-Krasovskii Functional in General Case}

We provide our general construction under the following assumption, where we use $\dot{V}(t)$ to mean $(d / d t) V(t, x(t))$ to make our notation concise:

Assumption 4 There exist a continuous functional $V$ : $[-\tau, \infty) \times C_{\text {in }} \rightarrow[0, \infty)$; functions $\alpha_{1}$ and $\alpha_{2}$ of class $\mathcal{K}_{\infty} ;$ a continuous nondecreasing function $\beta:[0, \infty) \rightarrow[0, \infty)$; a function $\gamma \in \mathcal{K}$; positive constants $\mu_{B}, \delta$, and $T$; and a continuous function $\mu: \mathbb{R} \rightarrow\left[-\mu_{B}, \mu_{B}\right]$ such that

$$
\alpha_{1}(|\phi(0)|) \leq V(t, \phi) \leq \alpha_{2}\left(|\phi|_{\infty}\right)
$$

holds for all $t \geq 0$ and $\phi \in C_{\mathrm{in}}$, such that

$$
\dot{V}(t) \leq \mu(t) V\left(t, x_{t}\right)+\beta\left(V\left(t, x_{t}\right)\right) V^{2}\left(t, x_{t}\right)+\gamma(|\xi(t)|)
$$

holds along all trajectories of the system (21), and such that

$$
\frac{1}{T} \int_{t-T}^{t} \mu(m) \mathrm{d} m \leq-\delta
$$

holds for all $t \in \mathbb{R}$.

Assumption 4 is significantly weaker than the usual requirement that $\mu$ must have a negative constant upper bound. In fact, $(25)$ says that $-\mu$ is a persistency of excitation parameter, but the existing literature on persistence of excitation did not solve the challenging problem of constructing Lyapunov-Krasovskii functionals that we solve in this section. Condition (25) says that $\mu$ is negative in a suitable averaged sense, and includes functions such as

$$
\mu(m)=-\max \{0, \sin (2 \pi t / T)\}
$$

and many other cases where $\mu$ can be zero throughout intervals of any positive length $L$. We prove the following result (but see Remark 2 for local ISS results for cases where $\beta$ is not necessarily zero):

Theorem 2 Let the system (21) satisfy Assumptions 34. If $\beta(m)=0$ for all $m \geq 0$, then (21) admits the ISS Lyapunov-Krasovskii functional

$$
\mathcal{W}(t, \phi)=e^{-\frac{1}{T} \int_{t-T}^{t} \int_{\ell}^{t} \mu(m) \mathrm{d} m \mathrm{~d} \ell} V(t, \phi)
$$

and therefore is ISS with respect to $\xi$.

Proof: In the sequel, all inequalities and equalities should be understood to hold along all trajectories of (21) and all $t \geq 0$, and we use the notation

$$
\dot{\mathcal{W}}(t)=\frac{d}{d t} \mathcal{W}\left(t, x_{t}\right)
$$

as we did for $V$. Since we are assuming that $\beta$ is identically equal to zero, we can use (24)-(25) to obtain

$$
\begin{aligned}
\dot{\mathcal{W}}(t)= & e^{-\frac{1}{T} \int_{t-T}^{t} \int_{\ell}^{t} \mu(m) \mathrm{d} m \mathrm{~d} \ell} \dot{V}(t) \\
& +\left[-\mu(t)+\frac{1}{T} \int_{t-T}^{t} \mu(m) \mathrm{d} m\right] \mathcal{W}\left(t, x_{t}\right) \\
\leq & e^{-\frac{1}{T} \int_{t-T}^{t} \int_{\ell}^{t} \mu(m) \mathrm{d} m \mathrm{~d} \ell} \gamma(|\xi(t)|) \\
& +\frac{1}{T} \int_{t-T}^{t} \mu(m) \mathrm{d} m \mathcal{W}\left(t, x_{t}\right) \\
\leq & -\delta \mathcal{W}\left(t, x_{t}\right) \\
& +e^{-\frac{1}{T} \int_{t-T}^{t} \int_{\ell}^{t} \mu(m) \mathrm{d} m \mathrm{~d} \ell} \gamma(|\xi(t)|) .
\end{aligned}
$$

Since $|\mu|$ is bounded by $\mu_{B}$, it follows that

$$
\dot{\mathcal{W}}(t) \leq-\delta \mathcal{W}\left(t, x_{t}\right)+e^{\frac{T}{2} \mu_{B}} \gamma(|\xi(t)|)
$$

holds for all $t \geq 0$, and (23) gives

$$
\begin{aligned}
& e^{-\frac{T}{2} \mu_{B}} \alpha_{1}(|\phi(0)|) \leq e^{-\frac{T}{2} \mu_{B}} V(t, \phi) \leq \mathcal{W}(t, \phi) \\
& \leq e^{\frac{T}{2} \mu_{B}} V(t, \phi) \leq e^{\frac{T}{2} \mu_{B}} \alpha_{2}\left(|\phi|_{\infty}\right)
\end{aligned}
$$

for all $t \geq 0$ and $\phi \in C_{\text {in }}$. Conditions (28)-(29) imply that $\mathcal{W}$ is an ISS Lyapunov-Krasovskii functional for (21). This proves the theorem.

Remark 2 When $\beta$ is present, we can prove local ISS results, as follows. Using (24) and the equality in (27), we deduce that

$$
\begin{aligned}
& \dot{\mathcal{W}}(t) \leq-\delta \mathcal{W}\left(t, x_{t}\right)+e^{\frac{T}{2} \mu_{B}} \gamma(|\xi(t)|) \\
& +e^{-\frac{1}{T} \int_{t-T}^{t} \int_{\ell}^{t} \mu(m) \mathrm{d} m \mathrm{~d} \ell} \beta\left(V\left(t, x_{t}\right)\right) V^{2}\left(t, x_{t}\right) .
\end{aligned}
$$

Since $|\mu|$ is bounded by our constant $\mu_{B}$ and $\beta$ is nondecreasing and $V\left(t, x_{t}\right) \leq e^{T \mu_{B} / 2} \mathcal{W}\left(t, x_{t}\right)$ holds for all $t$, we have

$$
\begin{aligned}
\dot{\mathcal{W}}(t) \leq[ & \left.-\delta+e^{\frac{3 T}{2} \mu_{B}} \beta\left(e^{\frac{T}{2} \mu_{B}} \mathcal{W}\left(t, x_{t}\right)\right) \mathcal{W}\left(t, x_{t}\right)\right] \\
& \times \mathcal{W}\left(t, x_{t}\right)+e^{\frac{T}{2} \mu_{B}} \gamma(|\xi(t)|) .
\end{aligned}
$$

Since $\gamma \in \mathcal{K}$, we can then choose a small enough constant $\mathcal{W}_{B}>0$ and then a small enough constant $\xi_{B}>0$ such that

$$
\begin{aligned}
& {\left[-\delta+e^{\frac{3 T}{2} \mu_{B}} \beta\left(e^{\frac{T}{2} \mu_{B}} \mathcal{W}_{B}\right) \mathcal{W}_{B}\right] \mathcal{W}_{B}} \\
& +e^{\frac{T}{2} \mu_{B}} \gamma\left(\xi_{B}\right)<0
\end{aligned}
$$


is satisfied. We now show that the ISS property is satisfied for all perturbations $\xi$ that are bounded in the supremum norm by $\xi_{B}$ and all initial functions $\phi \in C_{\mathrm{in}}$ such that

$$
\mathcal{W}(t, \phi) \leq \mathcal{W}_{B} \text { for all } t \geq 0 \text {. }
$$

(Notice that (33) holds if $|\phi|_{\infty} \leq \alpha_{2}^{-1}\left(\exp \left(-T \mu_{B} / 2\right) \mathcal{W}_{B}\right)$, by (29), where $|\cdot|_{\infty}$ is the usual supremum norm.)

To this end, we first observe that if the initial function $\phi$ satisfies (33), then (31) implies that

$$
\begin{aligned}
\dot{\mathcal{W}}(0) \leq & {\left[-\delta+e^{\frac{3 T}{2} \mu_{B}} \beta\left(e^{\frac{T}{2} \mu_{B}} \mathcal{W}_{B}\right) \mathcal{W}_{B}\right] \mathcal{W}_{B} } \\
& +e^{\frac{T}{2} \mu_{B}} \gamma\left(\xi_{B}\right) .
\end{aligned}
$$

From (32), it follows that $\dot{\mathcal{W}}(0)<0$. By continuity of $\mathcal{W}\left(t, x_{t}\right)$, we deduce that there is $t_{1}>0$ such that $\mathcal{W}\left(t, x_{t}\right)<$ $\mathcal{W}_{B}$ holds for all $t \in\left(0, t_{1}\right]$. Now we proceed by contradiction. Suppose that there were a $t_{2}>t_{1}$ such that $\mathcal{W}\left(t, x_{t}\right)<$ $\mathcal{W}_{B}$ for all $t \in\left[t_{1}, t_{2}\right)$ and $\mathcal{W}\left(t_{2}, x_{t_{2}}\right)=\mathcal{W}_{B}$. Then

$$
\begin{aligned}
\dot{\mathcal{W}}\left(t_{2}\right) \leq & {\left[-\delta+e^{\frac{3 T}{2} \mu_{B}} \beta\left(e^{\frac{T}{2} \mu_{B}} \mathcal{W}_{B}\right) \mathcal{W}_{B}\right] \mathcal{W}_{B} } \\
& +e^{\frac{T}{2} \mu_{B}} \gamma\left(\xi_{B}\right)<0 .
\end{aligned}
$$

Therefore there is $t_{3} \in\left(t_{1}, t_{2}\right)$ such that $\mathcal{W}\left(t_{3}, x_{t_{3}}\right)>$ $\mathcal{W}\left(t_{2}, x_{t_{2}}\right)=\mathcal{W}_{B}$. This yields a contradiction.

It follows from (31) that for all $t \geq 0$, we have

$$
\begin{aligned}
& \dot{\mathcal{W}}(t) \leq-\bar{\lambda} \mathcal{W}\left(t, x_{t}\right)+e^{\frac{T}{2} \mu_{B}} \gamma(|\xi(t)|), \text { where } \\
& \bar{\lambda}=\delta-e^{\frac{3 T}{2} \mu_{B}} \beta\left(e^{\frac{T}{2} \mu_{B}} \mathcal{W}_{B}\right) \mathcal{W}_{B} .
\end{aligned}
$$

Notice for later use that (32) implies that $\bar{\lambda}>0$. By integrating (36) on any interval $\left[t_{0}, t\right]$, and using (29), we obtain the ISS inequality

$$
\begin{aligned}
|x(t)| \leq & \alpha_{1}^{-1}\left(2 e^{-\bar{\lambda}\left(t-t_{0}\right)} e^{T \mu_{B}} \alpha_{2}\left(\left|x_{t_{0}}\right|_{\infty}\right)\right) \\
& +\alpha_{1}^{-1}\left(\frac{2 e^{T \mu_{B}}}{\lambda} \sup _{\ell \in\left[t_{0}, t\right]} \gamma(|\xi(\ell)|)\right),
\end{aligned}
$$

where we also used the fact that the function $\alpha_{1}^{-1} \in \mathcal{K}_{\infty}$ satisfies $\alpha_{1}^{-1}(a+b) \leq \alpha_{1}^{-1}(2 a)+\alpha_{1}^{-1}(2 b)$ for all $a \geq 0$ and $b \geq 0$. This proves the local ISS result.

\subsection{System with Pointwise and Distributed Delays}

Theorem 2 requires a function $V$ that satisfies Assumption 4. In this section, we study the system (21) under assumptions which are frequently met in practice and again show how Lyapunov-Krasovskii functionals can be constructed. Our first assumption is:

Assumption 5 There are a constant $\eta>0$, a piecewise $C^{1}$ Lyapunov function $\mathcal{V}:[-\max \{\tau, \eta\}, \infty) \times \mathbb{R}^{n} \rightarrow[0, \infty)$, functions $\alpha_{1}$ and $\alpha_{2}$ of class $\mathcal{K}_{\infty}$, a function $\gamma \in \mathcal{K}$, a constant $\omega \in[0, \eta]$, a bounded continuous function $a: \mathbb{R} \rightarrow$ $\mathbb{R}$, and bounded continuous functions $b: \mathbb{R} \rightarrow[0, \infty)$ and $c: \mathbb{R}^{2} \rightarrow[0, \infty)$ such that

$$
\left.\alpha_{1}(\mid x) \mid\right) \leq \mathcal{V}(t, x) \leq \alpha_{2}(|x|)
$$

holds for all $t \geq 0$ and $x \in \mathbb{R}^{n}$ and such that along all trajectories of the system (21), we have

$$
\begin{aligned}
& \dot{\mathcal{V}}(t) \leq a(t) \mathcal{V}(t, x(t))+b(t) \mathcal{V}(t-\tau, x(t-\tau)) \\
& +\int_{t-\eta}^{t-\omega} c(m, t) \mathcal{V}(m, x(m)) \mathrm{d} m+\gamma(|\xi(t)|)
\end{aligned}
$$

almost everywhere.

In terms of the functions from Assumption 5, we then set

$$
\begin{aligned}
& h(t)= \\
& e^{-\int_{t}^{t+\tau} a(\ell) \mathrm{d} \ell} b(t+\tau)+\int_{t+\omega}^{t+\eta} e^{-\int_{t}^{s} a(\ell) \mathrm{d} \ell} c(t, s) \mathrm{d} s
\end{aligned}
$$

and add this assumption:

Assumption 6 There are constants $T>0$ and $\delta>0$ such that

holds for all $t \geq 0$.

$$
\frac{1}{T} \int_{t-T}^{t}[a(m)+h(m)] \mathrm{d} m \leq-\delta
$$

Condition (39) implies (4) from Assumption 1 when the perturbation $\xi$ is the zero function, so (39) is more restrictive than (4). However the functions $b$ and $c$ in (39) will be useful for building a Lyapunov-Krasovskii functional; see (47). Hence, this section adds considerable value, as compared with Section 3. To build our Lyapuov-Krasovskii functional for (21), we introduce the function

$$
q(m)=\int_{m-\eta}^{m-\omega} e^{\int_{s+\eta}^{m} \lambda(\ell) \mathrm{d} \ell} c(m-\omega, s+\eta) \mathrm{d} s
$$

and the operators

$$
\begin{gathered}
\Gamma_{1}\left(t, x_{t}\right)=\int_{t-\tau}^{t} e^{\int_{m+\tau}^{t} \lambda(\ell) \mathrm{d} \ell} b(m+\tau) \mathcal{V}(m, x(m)) \mathrm{d} m, \\
\Gamma_{2}\left(t, x_{t}\right)= \\
\int_{t-\omega}^{t} e^{\int_{m+\omega}^{t} \lambda(\ell) \mathrm{d} \ell} q(m+\omega) \mathcal{V}(m, x(m)) \mathrm{d} m, \text { and } \\
\Gamma_{3}\left(t, x_{t}\right)=\int_{t-\eta}^{t-\omega} e^{\int_{m+\eta}^{t} \lambda(\ell) \mathrm{d} \ell} \mathcal{G}(t, \omega, m) \mathrm{d} m,
\end{gathered}
$$

where $\lambda=a+h$ and

$$
\mathcal{G}(t, \omega, m)=\int_{m}^{t-\omega} c(s, m+\eta) \mathcal{V}(s, x(s)) \mathrm{d} s .
$$

We prove the following result (but see Remark 3 for local versions under weaker conditions, and generalizations with several types of delays, and see below for illustrations):

Corollary 1 Let the system (21) satisfy Assumptions 3 and 5-6. Then the system is ISS with respect to $\xi$, and

$$
\begin{aligned}
& \mathcal{W}\left(t, x_{t}\right)=e^{-\frac{1}{T} \int_{t-T}^{t} \int_{\ell}^{t} \lambda(m) \mathrm{d} m \mathrm{~d} \ell} \mathcal{U}\left(t, x_{t}\right) \\
& \text { with the choice } \mathcal{U}\left(t, x_{t}\right)= \\
& \mathcal{V}(t, x(t))+\Gamma_{1}\left(t, x_{t}\right)+\Gamma_{2}\left(t, x_{t}\right)+\Gamma_{3}\left(t, x_{t}\right)
\end{aligned}
$$

is an ISS Lyapunov-Krasovskii functional for (21).

Proof: Throughout the proof, we let the function $\xi$ be equal to zero. The general case follows by adding $\gamma(|\xi(t)|)$ to the right sides of the relevant inequalities in the rest of the 
proof. First note that

$$
\begin{aligned}
\dot{\Gamma}_{1}(t)= & \lambda(t) \Gamma_{1}\left(t, x_{t}\right)+e^{\int_{t+\tau}^{t} \lambda(\ell) \mathrm{d} \ell} b(t+\tau) \mathcal{V}(t, x(t)) \\
& -b(t) \mathcal{V}(t-\tau, x(t-\tau)), \\
\dot{\Gamma}_{2}(t)= & \lambda(t) \Gamma_{2}\left(t, x_{t}\right)+e^{\int_{t+\omega}^{t} \lambda(\ell) \mathrm{d} \ell} q(t+\omega) \mathcal{V}(t, x(t)) \\
& -q(t) \mathcal{V}(t-\omega, x(t-\omega))
\end{aligned}
$$

and

$$
\begin{aligned}
& \dot{\Gamma}_{3}(t)=\lambda(t) \Gamma_{3}\left(t, x_{t}\right)-\int_{t-\eta}^{t-\omega} c(s, t) \mathcal{V}(s, x(s)) \mathrm{d} s \\
& +\int_{t-\eta}^{t-\omega} e^{\int_{m+\eta}^{t} \lambda(\ell) \mathrm{d} \ell} c(t-\omega, m+\eta) \mathcal{V}(t-\omega, x(t-\omega)) \mathrm{d} m \\
& =\lambda(t) \Gamma_{3}\left(t, x_{t}\right)-\int_{t-\eta}^{t-\omega} c(s, t) \mathcal{V}(s, x(s)) \mathrm{d} s \\
& +q(t) \mathcal{V}(t-\omega, x(t-\omega))
\end{aligned}
$$

hold along all trajectories of the system. Using Assumption 5 and summing the right sides of the preceding equalities for the $\dot{\Gamma}_{i}$ 's for $i=1,2,3$ and then combining terms, it follows that the derivative of $\mathcal{U}$ along all trajectories of (21) satisfies

$$
\begin{aligned}
\dot{\mathcal{U}}(t) \leq & a(t) \mathcal{V}(t, x(t))+b(t) \mathcal{V}(t-\tau, x(t-\tau)) \\
& +\int_{t-\eta}^{t-\omega} c(m, t) \mathcal{V}(m, x(m)) \mathrm{d} m \\
& +\dot{\Gamma}_{1}(t)+\dot{\Gamma}_{2}(t)+\dot{\Gamma}_{3}(t) \\
= & {\left[a(t)+e^{\int_{t+\tau}^{t} \lambda(\ell) \mathrm{d} \ell} b(t+\tau)\right.} \\
& \left.+e^{\int_{t+\omega}^{t} \lambda(\ell) \mathrm{d} \ell} q(t+\omega)\right] \mathcal{V}(t, x(t)) \\
& +\lambda(t)\left[\Gamma_{1}\left(t, x_{t}\right)+\Gamma_{2}\left(t, x_{t}\right)+\Gamma_{3}\left(t, x_{t}\right)\right] .
\end{aligned}
$$

We now use the functions

$$
\begin{aligned}
& b_{*}(t)=e^{-\int_{t}^{t+\tau} a(\ell) \mathrm{d} \ell} b(t+\tau) \text { and } \\
& c_{*}(t, s)=e^{\int_{s+\eta}^{t} a(\ell) \mathrm{d} \ell} c(t, s+\eta) .
\end{aligned}
$$

Since $b$ and $c$ are nonnegative valued, it follows that $b_{*}$ and $c_{*}$ are nonnegative valued. Also, since $\lambda=a+h$, the estimates (48) give

$$
\begin{aligned}
\dot{\mathcal{U}}(t) \leq & a(t) \mathcal{U}\left(t, x_{t}\right)+\left[e^{\int_{t+\tau}^{t}(a(\ell)+h(\ell)) \mathrm{d} \ell} b(t+\tau)\right. \\
& \left.+e^{\int_{t+\omega}^{t}(a(\ell)+h(\ell)) \mathrm{d} \ell} q(t+\omega)\right] \mathcal{V}(t, x(t)) \\
& +h(t)\left[\Gamma_{1}\left(t, x_{t}\right)+\Gamma_{2}\left(t, x_{t}\right)+\Gamma_{3}\left(t, x_{t}\right)\right] .
\end{aligned}
$$

Using the definitions of $q, b_{*}$, and $c_{*}$, we get

$$
\begin{aligned}
\dot{\mathcal{U}}(t) \leq & a(t) \mathcal{U}\left(t, x_{t}\right)+\left[e^{-\int_{t}^{t+\tau} h(\ell) \mathrm{d} \ell} b_{*}(t)\right. \\
& \left.+\int_{t+\omega-\eta}^{t} e^{-\int_{t}^{s+\eta} h(\ell) \mathrm{d} \ell} c_{*}(t, s) \mathrm{d} s\right] \mathcal{V}(t, x(t)) \\
& +h(t)\left[\Gamma_{1}\left(t, x_{t}\right)+\Gamma_{2}\left(t, x_{t}\right)+\Gamma_{3}\left(t, x_{t}\right)\right]
\end{aligned}
$$

and

$$
h(t)=b_{*}(t)+\int_{t+\omega-\eta}^{t} c_{*}(t, s) \mathrm{d} s .
$$

Since $h$ is nonnegative valued, we get

$$
\begin{aligned}
& e^{-\int_{t}^{t+\tau} h(\ell) \mathrm{d} \ell} b_{*}(t)+\int_{t+\omega-\eta}^{t} e^{-\int_{t}^{s+\eta} h(\ell) \mathrm{d} \ell} c_{*}(t, s) \mathrm{d} s \\
& \leq h(t) .
\end{aligned}
$$

As an immediate consequence, we get

$$
\dot{\mathcal{U}}(t) \leq[a(t)+h(t)] \mathcal{U}\left(t, x_{t}\right) .
$$

Hence, using Assumption 6 and Theorem 2 with $\beta=0$, we can conclude.

Remark 3 We can prove a local result when extra nonlinear terms are present in (39) as we did in Remark 2. We can also extend Theorem 1 to cases where several pointwise and distributed delays are present.

\subsection{Comparison of Approaches}

The purpose of this subsection is to compare the LyapunovKrasovskii functional construction method from the preceding proof with a possible alternative approach to building Lyapunov-Krasovskii functionals, and to show why the alternative approach cannot be used. This will further illustrate the value of our new approaches.

To this end, assume again that (21) satisfies Assumption 3, and that there are a $C^{1}$ function $V:[-\tau, \infty) \times \mathbb{R}^{n} \rightarrow[0, \infty)$ and functions $\alpha_{1}$ and $\alpha_{2}$ of class $\mathcal{K}_{\infty}$ such that $\alpha_{1}(|x|) \leq$ $V(t, x) \leq \alpha_{2}(|x|)$ hold for all $t \geq 0$ and $x \in \mathbb{R}^{n}$ and such that along the trajectories of $(2 \overline{1})$ with $\xi \equiv 0$, we have

$$
\dot{V}(t) \leq a(t) V(t, x(t))+b(t) V(t-\tau, x(t-\tau))
$$

where $a$ is a periodic function of some period $T>0$, the function $b$ is bounded and nonnegative valued, and both $a$ and $b$ are continuous. We also assume that there is a positive constant $a_{c}$ such that

$$
\frac{1}{T} \int_{0}^{T} a(m) \mathrm{d} m=-a_{c} .
$$

In this context, a first possible approach to establish stability conditions for (21) consists of the following strictification of the function $V$ with respect to $a$.

We define the function

$$
\begin{aligned}
& \nu_{1}(t, x)=e^{R(t)} V(t, x), \text { where } \\
& R(t)=-\frac{1}{T} \int_{t-T}^{t}\left(\int_{\ell}^{t} a(m) \mathrm{d} m\right) \mathrm{d} \ell .
\end{aligned}
$$

Since $\dot{R}(t)=-a(t)-a_{c}$, it follows that the time derivative of $\nu_{1}$ along all trajectories of (21) satisfies

$$
\begin{aligned}
\dot{\nu}_{1}(t)= & e^{R(t)} \dot{V}(t)+\dot{R}(t) e^{R(t)} V(t, x(t)) \\
\leq & (a(t)+\dot{R}(t)) \nu_{1}(t, x(t)) \\
& +e^{R(t)} b(t) V(t-\tau, x(t-\tau)) \\
\leq & -a_{c} \nu_{1}(t, x(t)) \\
& +e^{R(t)-R(t-\tau)} b(t) \nu_{1}(t-\tau, x(t-\tau)) .
\end{aligned}
$$

We deduce from Razumikhin's theorem that if there is a 
constant $s_{c}>0$ such that

$$
-a_{c}+e^{R(t)-R(t-\tau)} b(t) \leq-s_{c}
$$

for all $t$, then the origin of (21) with $\xi \equiv 0$ is globally asymptotically stable.

If we now wish to construct a Lyapunov-Krasovskii functional, then we can proceed as follows. Let

$$
\begin{aligned}
& \nu_{2}\left(t, x_{t}\right)=\nu_{1}(t, x(t))+ \\
& \int_{t-\tau}^{t} e^{R(m+\tau)-R(m)} b(m+\tau) \nu_{1}(m, x(m)) \mathrm{d} m .
\end{aligned}
$$

Then for all $t \geq 2 \tau$, we have

$$
\dot{\nu}_{2}(t) \leq\left(-a_{c}+e^{R(t+\tau)-R(t)} b(t+\tau)\right) \nu_{1}(t, x(t)) .
$$

Using the Lyapunov-Krasovskii theorem and (58), one can deduce from (60) that the system (21) is globally uniformly asymptotically stable. If $\tau=0$, then

$$
-a_{c}+e^{R(t+\tau)-R(t)} b(t+\tau)=-a_{c}+b(t)
$$

We deduce by continuity that when there are instants $t$ such that $b(t)>a_{c}$, global asymptotic stability for arbitrarily small delay cannot be deduced from (60). By contrast, our assumptions allow cases where $b(t)>a_{c}$ for some values $t$, so our results are less conservative; see the examples below.

\section{Discrete Time Versions}

In this section, we provide discrete time analogs of some of the continuous time ideas from the previous sections. The work in this section builds on the Razumikhin-like theorems in Elaydi and Zhang (1994) and Zhou (2014), by developing nonstrict Lyapunov-like decay conditions that imply exponential convergence properties. Discrete time analogs of the decay conditions on a continuous time Lyapunov function involve a sequence of values $\{V(x(k))\}$ taken by a nonnegative valued function $V$ along sequences $\{x(k)\}$ of state values of a delay system of the form

$$
x(k+1)=f(k, x(k-\tau), \ldots, x(k)) .
$$

Setting $V_{k}=V(x(k))$ for all $k \in \mathbb{N}$ leads to the study of sequences $\left\{V_{k}\right\}$ that are not necessarily decreasing in $k$, where $\mathbb{N}=\{1,2, \ldots\}$. Such sequences are the subject of this section.

Discrete time systems naturally arise from discretizing continuous time systems in control applications. Moreover, as in the continuous time case, time-varying discrete time systems naturally arise from tracking problems and from linearizing around a reference trajectory, even if the original system is time invariant, which is an important motivation for our allowing time-varying systems. To see how time-varying systems arise from tracking problems in a special case, assume that we are given a discrete time system $q(k+1)=\mathcal{F}(q(k))+u(q(k-\tau))$ with a control $u$ and a constant delay $\tau$, and a reference trajectory $q_{r}$ such that $q_{r}(k+1)=\mathcal{F}\left(q_{r}(k)\right)$ for all $k$ that we wish to track. Then the dynamics of the tracking error $x(k)=q(k)-q_{r}(k)$ are $x(k+1)=f(k, x(k-\tau), x(k))$ where $f(a, b, c)=$ $\mathcal{F}\left(c+q_{r}(a)\right)-\mathcal{F}\left(q_{r}(a)\right)+u\left(b+q_{r}(a-\tau)\right)$.

\subsection{Preliminary Result for Scalar Sequences}

To prove our discrete time results, we use:

Lemma 1 Let $\xi_{1}, \xi_{2}, \ldots$ be any sequence of positive real numbers such that there are constants $s_{1}>0$ and $s_{2} \geq s_{1}$ and an integer $p \geq 1$ for which $(i) \xi_{i} \in\left[s_{1}, s_{2}\right]$ for all $i \geq 1$ and (ii) the constant

$$
\sigma=\left(\prod_{i=1+j}^{p+j} \xi_{i}\right)^{\frac{1}{p}}
$$

is independent of the integer $j \geq 0$ and satisfies $\sigma \in(0,1)$. Then the sequence $\rho_{1}, \rho_{2}, \ldots$ defined by

$$
\rho_{k}=\prod_{i=1}^{k-1} \frac{\sigma}{\xi_{i}}
$$

for all $k \geq 2$ and $\rho_{1}=1$ satisfies $\left(\sigma / s_{2}\right)^{p+1} \leq \rho_{k} \leq$ $\left(\sigma / s_{1}\right)^{p+1}$ for all $k \geq 1$.

Proof: If $k$ is any integer such that $k \geq p+2$, and if we set $\ell=\operatorname{Floor}((k-2) / p)$, then the fact that $\sigma$ in (62) is independent of $j$, and the fact that $k-1 \leq(\ell+1) p+1$, give

$$
\rho_{k}=\left(\prod_{i=1}^{\ell p} \frac{\sigma}{\xi_{i}}\right)\left(\prod_{i=\ell p+1}^{k-1} \frac{\sigma}{\xi_{i}}\right)=\prod_{i=\ell p+1}^{k-1} \frac{\sigma}{\xi_{i}} .
$$

The last product in (64) lies in $\left[\left(\sigma / s_{2}\right)^{p+1},\left(\sigma / s_{1}\right)^{p+1}\right]$ because our assumption that $\xi_{i} \in\left[s_{1}, s_{2}\right]$ for all $i$ implies that $\sigma \in\left[s_{1}, s_{2}\right]$, so $\sigma / s_{2} \leq 1 \leq \sigma / s_{1}$. Since we can check directly that $\rho_{k} \in\left[\left(\sigma / s_{2}\right)^{p+1},\left(\sigma / s_{1}\right)^{p+1}\right]$ for all $k \in$ $\{1,2, \ldots, p+1\}$, the lemma follows.

Before turning to our main result for discrete time cases, we present a discrete time strictification result, which is of interest for its own sake and eases the understanding of the main result.

Proposition 1 Let $\left\{\xi_{k}\right\}$ and the constants $\sigma \in(0,1), s_{1}>$ 0 , and $s_{2} \geq s_{1}$ satisfy the assumptions of Lemma 1, and $\left\{V_{k}\right\}$ be a sequence of nonnegative real numbers such that

$$
V_{k+1} \leq \xi_{k} V_{k}
$$

holds for all $k \geq 1$. Then $V_{k} \leq\left(s_{2} / s_{1}\right)^{p+1} \sigma^{k-1} V_{1}$ holds for all $k \geq 1$, so $\left\{\bar{V}_{k}\right\}$ is exponentially stable.

Proof: We use the sequence $\mathcal{Q}_{k}=\rho_{k} V_{k}$, where $\left\{\rho_{k}\right\}$ is from (63) in Lemma 1. Then the fact that $\rho_{k+1} / \rho_{k}=\sigma / \xi_{k}$ for all $k \geq 1$ implies that for all $k \geq 1$, we obtain

$$
\mathcal{Q}_{k+1}=\rho_{k+1} V_{k+1} \leq \rho_{k+1} \xi_{k} V_{k}=\frac{\rho_{k+1}}{\rho_{k}} \xi_{k} \mathcal{Q}_{k}=\sigma \mathcal{Q}_{k}
$$

and so also $\mathcal{Q}_{k} \leq \sigma^{k-1} \mathcal{Q}_{1}$. The result now follows from the positive bounds for $\left\{\rho_{k}\right\}$ from Lemma 1 .

Remark 4 The assumptions of Proposition 1 do not imply that $\xi_{k} \leq 1$ for all $k \in \mathbb{N}$. The proof of Proposition 1 relies on a strictification approach, and ensures exponential stability. An alternative proof of the exponential stability conclusion consists of observing that since $\sigma \in(0,1)$ in (62) is 
independent of $j$, it follows from (65) that for all $k \geq p$, we can choose $j=k-p$ in (62) to get

$$
\begin{aligned}
& V_{k+1} \leq \xi_{k} V_{k} \leq \prod_{i=k-p+1}^{k} \xi_{i} V_{k-p+1} \\
& =\sigma^{p} V_{k-p+1} \leq \sigma V_{k-p+1} .
\end{aligned}
$$

Hence, for each constant $g \in\{1, \ldots, p-1\}$, the sequence $\left\{V_{k p+g}\right\}$ is exponentially stable. We deduce that $\left\{V_{k}\right\}$ is exponentially stable. In fact, for all $k \geq p+1$, we can choose $\ell=\operatorname{Floor}((k-1) / p)$ and use the facts that $\xi_{i} \leq s_{2}$ for all $i$ and $\sigma<1$ to get

$$
\begin{aligned}
& V_{k} \leq \prod_{i=\ell p}^{k-1} \xi_{i} V_{\ell p} \leq \prod_{i=\ell p}^{k-1} \xi_{i} \sigma^{\ell-1} V_{p} \leq \\
& \left(\prod_{i=\ell p}^{k-1} \xi_{i}\right)\left(\prod_{i=1}^{p-1} \xi_{i}\right) \sigma^{\ell-1} V_{1} \\
& \leq \max \left\{s_{2}^{2 p}, 1\right\} \sigma^{\text {Floor }((k-1) / p)-1} V_{1},
\end{aligned}
$$

by separately considering the cases where $s_{2} \geq 1$ and $s_{2}<1$.

\subsection{Main Result for Discrete Time Case}

The section provides a discrete-time version of results of the previous sections. However, it is not a strictified version of the Razumikhin theorem for discrete time systems but is an extension of Proposition 1 that allows delays.

Consider a sequence of nonnegative real numbers $\left\{V_{k}\right\}$ such that there exist an integer $p>0$ and sequences $\left\{\alpha_{k, j}\right\}$ of nonnegative values for all $j \in\{0, \ldots, p\}$ such that for all $k>p$, the following inequality is satisfied:

$$
V_{k+1} \leq \sum_{j=0}^{p} \alpha_{k, j} V_{k-j}
$$

For instance, the $V_{k}$ 's can be the values taken by a Lyapunov function at discrete times. We define the sequence

$$
\bar{\alpha}_{k}=\sum_{j=0}^{p} \alpha_{k, j}
$$

and make the following two assumptions:

Assumption 7 There are constants $a_{1}>0$ and $a_{2} \geq a_{1}$ such that $\bar{\alpha}_{k} \in\left[a_{1}, a_{2}\right]$ holds for all $k \in \mathbb{N}$. Also,

$$
\alpha_{*}=\left(\prod_{i=1+j}^{p+j} \bar{\alpha}_{i}\right)^{\frac{1}{p}}
$$

is independent of the integer $j \geq 0$ and satisfies $\alpha_{*} \in(0,1)$.

Assumption 8 The inequality $\alpha_{*}^{p+1}<a_{1}^{p}$ is satisfied.

We can then prove this exponential stability result:

Theorem 3 If $\left\{V_{k}\right\}$ is such that Assumptions 7-8 hold, then we can find a constant $\bar{\lambda} \in(0,1)$ such that

$$
V_{k+1} \leq\left(\frac{a_{2}}{a_{1}}\right)^{p+1} \bar{\lambda}^{k-p-1} \sup _{\{i=1, \ldots, p+1\}} V_{i}
$$

holds for all $k>p$.
Proof: We define the sequence $\lambda_{k}$ by

$$
\lambda_{1}=1 \quad \text { and } \quad \lambda_{k}=\prod_{i=1}^{k-1} \frac{\alpha_{*}}{\bar{\alpha}_{i}} \quad \text { when } \quad k>1
$$

and the sequence $W_{k}=\lambda_{k} V_{k}$. Then for all $k>p$, we can use our inequality (68) to get

$$
\begin{aligned}
W_{k+1} & \leq \sum_{j=0}^{p} \alpha_{k, j} \frac{\lambda_{k+1}}{\lambda_{k-j}} W_{k-j} \\
& =\frac{\lambda_{k+1}}{\lambda_{k}} \sum_{j=0}^{p} \alpha_{k, j} \frac{\lambda_{k}}{\lambda_{k-j}} W_{k-j} \\
& \leq \frac{\lambda_{k+1}}{\lambda_{k}} \sum_{j=0}^{p} \alpha_{k, j} \frac{\lambda_{k}}{\lambda_{k-j}} \sup _{i \in\{0, \ldots, p\}} W_{k-i} \\
& \leq \frac{\lambda_{k+1}}{\lambda_{k}} \bar{\alpha}_{k} \sup _{i \in\{k-p, \ldots, k\}} \frac{\lambda_{k}}{\lambda_{i}} \sup _{i \in\{k-p, \ldots, k\}} W_{i} .
\end{aligned}
$$

From (72), it follows that for all $k>p$, we have

$$
\begin{aligned}
& W_{k+1} \leq \frac{\alpha_{*}}{\bar{\alpha}_{k}} \bar{\alpha}_{k} \sup _{i \in\{k-p, \ldots, k\}} \frac{\lambda_{k}}{\lambda_{i}} \sup _{i \in\{k-p, \ldots, k\}} W_{i} \\
& =\alpha_{*} \max \left\{\sup _{i \in\{k-p, \ldots, k-1\}} \prod_{m=i}^{k-1} \frac{\alpha_{*}}{\bar{\alpha}_{m}}, 1\right\} \sup _{i \in\{k-p, \ldots, k\}} W_{i} .
\end{aligned}
$$

Using the lower bound $a_{1}$ for the sequence $\bar{\alpha}_{m}$, we obtain

$$
W_{k+1} \leq\left[\alpha_{*} \max \left\{1,\left(\frac{\alpha_{*}}{a_{1}}\right)^{p}\right\}\right] \sup _{i \in\{k-p, \ldots, k\}} W_{i},
$$

by separately considering the cases $\alpha_{*} \leq a_{1}$ and $\alpha_{*}>a_{1}$. Let $\bar{\lambda}$ be the quantity in squared brackets in (74). Then Assumptions $7-8$ imply that $\bar{\lambda} \in(0,1)$, and (74) gives

$$
W_{k+1} \leq \bar{\lambda}^{k-p-1} \sup _{\{i=1, \ldots, p+1\}} W_{i}
$$

If we now apply Lemma 1 with the choices $\xi_{i}=\bar{\alpha}_{i}, s_{1}=a_{1}$, $s_{2}=a_{2}$, and $\sigma=\alpha_{*}$, then we get

$$
\left(\frac{\alpha_{*}}{a_{2}}\right)^{p+1} \leq \lambda_{i} \leq\left(\frac{\alpha_{*}}{a_{1}}\right)^{p+1}
$$

for all $i$, so the desired decay estimate (71) follows from our formula $W_{k}=\lambda_{k} V_{k}$ for the $W_{k}$ 's.

\section{Periodic Case}

\subsection{Statement of Result for Periodic Case}

We now consider key cases where the following holds:

Assumption 9 Assumption 1 holds, and there is a constant $\mathcal{T}>0$ such that $a$ and $b$ have period $\mathcal{T}$. Also, there exists a bounded piecewise continuous function $\epsilon: \mathbb{R} \rightarrow \mathbb{R}$ that is periodic of period $\mathcal{T}$ and that satisfies

$$
\int_{0}^{\mathcal{T}}[\epsilon(m)+a(m)+b(m)] \mathrm{d} m=0
$$

and is such that the function $\kappa$ defined in (7) is such that

$$
\sup _{t \in[0, \mathcal{T}]}\left[\left(e^{\kappa(t)}-1\right) b(t)-\epsilon(t)\right]<0
$$

is satisfied. 
In Section 6.3, we prove:

Corollary 2 Let (2) satisfy Assumption 9. Then the origin of (2) is globally uniformly asymptotically stable.

\subsection{Discussion of Corollary 2}

Assumption 9 is often satisfied. For instance, consider the case where $a$ and $b$ have some period $\mathcal{T}$ and choose the constant function $\epsilon(t)=\epsilon_{*}$, where

$$
\epsilon_{*}=-\frac{1}{\mathcal{T}} \int_{0}^{\mathcal{T}} \mu(\ell) \mathrm{d} \ell
$$

and $\mu=a+b$ as before. Then the function (7) satisfies

$$
\kappa(t)=\sup _{\ell \in[t-\tau, t]} \int_{\ell}^{t}\left(-\epsilon_{*}-\mu(s)\right) \mathrm{d} s \leq \tau s_{\mu}
$$

for all $t \geq 0$, where $s_{\mu}=\sup _{s \in[0, \mathcal{T}]}\left(-\epsilon_{*}-\mu(s)\right)$, if $s_{\mu} \geq 0$, and $\kappa(t) \leq 0$ if $s_{\mu} \leq 0$. Assume that $\epsilon_{*}>0$ and $s_{\mu} \geq 0$. Then (76)-(77) hold if the inequality

$$
\sup _{t \in[0, \mathcal{T}]}\left[\left(e^{\tau s_{\mu}}-1\right) b(t)\right]<\epsilon_{*}
$$

is satisfied. If $s_{\mu}>0$, then (80) holds if $\tau \in\left(0, \tau_{*}\right)$, where

$$
\tau_{*}=\frac{1}{s_{\mu}} \ln \left(1+\frac{\epsilon_{*}}{\bar{b}}\right) \text {. }
$$

Another sufficient condition can be obtained when $\mu$ is globally Lipschitz, as follows. Suppose that we have a global Lipschitz constant $\lambda_{\mu}>0$ for $\mu$. For all $s \geq 0$, we get

$$
\epsilon_{*}=-\frac{1}{\mathcal{T}} \int_{s-\mathcal{T}}^{s} \mu(\ell) \mathrm{d} \ell,
$$

by the periodicity of $a$ and $b$. Hence,

$$
\kappa(t)=\sup _{\ell \in[t-\tau, t]} \int_{\ell}^{t}\left(\frac{1}{\mathcal{T}} \int_{s-\mathcal{T}}^{s} \mu(m) \mathrm{d} m-\mu(s)\right) \mathrm{d} s,
$$

by our choice $\epsilon(t)=\epsilon_{*}$. Since the function

$$
\varphi(t)=\frac{1}{\mathcal{T}} \int_{t-\mathcal{T}}^{t} \int_{s}^{t} \mu(m) \mathrm{d} m \mathrm{~d} s
$$

satisfies

$$
\dot{\varphi}(t)=\mu(t)-\frac{1}{\mathcal{T}} \int_{t-\mathcal{T}}^{t} \mu(m) \mathrm{d} m
$$

we can integrate $\dot{\varphi}$ over $[\ell, t]$ to get

$$
\begin{aligned}
\kappa(t) & =\sup _{\ell \in[t-\tau, t]} \int_{\ell}^{t}(-\dot{\varphi}(s)) \mathrm{d} s \\
& =\sup _{\ell \in[t-\tau, t]}(-\varphi(t)+\varphi(\ell)) .
\end{aligned}
$$

Since we can change variables to get

$$
\varphi(\ell)=\frac{1}{\mathcal{T}} \int_{t-\mathcal{T}}^{t}\left(\int_{s}^{t} \mu(m-t+\ell) \mathrm{d} m\right) \mathrm{d} s,
$$

our Lipschitz constant $\lambda_{\mu}>0$ and (86)-(87) give

$$
\begin{aligned}
& \kappa(t)= \\
& \sup _{\ell \in[t-\tau, t]} \frac{1}{\mathcal{T}} \int_{t-\mathcal{T}}^{t}\left(\int_{s}^{t}[-\mu(m)+\mu(m-t+\ell)] \mathrm{d} m\right) \mathrm{d} s \\
& \leq \sup _{\ell \in[t-\tau, t]} \frac{\lambda_{\mu}}{\mathcal{T}}\left(\int_{t-\mathcal{T}}^{t} \int_{s}^{t}(t-\ell) \mathrm{d} \ell\right) \mathrm{d} s \leq \frac{\mathcal{T} \lambda_{\mu} \tau}{2},
\end{aligned}
$$

by upper bounding the last integrand by $\tau$. Hence, (76)(77) from Assumption 9 are satisfied if $\epsilon_{*}>0$ and

$$
\left(e^{\frac{\tau \lambda_{\mu} \tau}{2}}-1\right) \bar{b}<\epsilon_{*}
$$

are satisfied. This leads to the delay dependent condition

$$
\lambda_{\mu} \tau \mathcal{T}<2 \ln \left(1+\frac{\epsilon_{*}}{\bar{b}}\right)
$$

\subsection{Proof of Corollary 2}

We show that Assumption 9 implies that Assumption 2 holds. Let $\bar{\epsilon}>0, \bar{a}>0$, and $\bar{b}>0$ be bounds on the functions $|\epsilon|,|a|$, and $b$, respectively, $t>0$ be given, and $k$ be the integer such that $t \in[k \mathcal{T},(k+1) \mathcal{T})$. Then

$$
\begin{aligned}
& \left|\int_{0}^{t}(\epsilon(\ell)+\mu(\ell)) \mathrm{d} \ell\right| \leq\left|\int_{0}^{k \mathcal{T}}(\epsilon(\ell)+\mu(\ell)) \mathrm{d} \ell\right| \\
& +\left|\int_{k \mathcal{T}}^{t}(\epsilon(\ell)+\mu(\ell)) \mathrm{d} \ell\right| .
\end{aligned}
$$

Since (76) and our choice $\mu=a+b$ imply that

$$
\int_{0}^{k \mathcal{T}}(\epsilon(\ell)+\mu(\ell)) \mathrm{d} \ell=0,
$$

it follows from (90) that

$$
\begin{aligned}
& \left|\int_{0}^{t}(\epsilon(\ell)+\mu(\ell)) \mathrm{d} \ell\right| \leq \int_{k \mathcal{T}}^{t}(\bar{\epsilon}+\bar{a}+\bar{b}) \mathrm{d} \ell \\
& \leq \mathcal{T}(\bar{\epsilon}+\bar{a}+\bar{b})
\end{aligned}
$$

Next, we prove that $\kappa$ is periodic of period $\mathcal{T}$. We have

$$
\begin{aligned}
\kappa(t+\mathcal{T}) & =\sup _{\ell \in[t+\mathcal{T}-\tau, t+\mathcal{T}]} \int_{\ell}^{t+\mathcal{T}}(-\epsilon(m)-\mu(m)) \mathrm{d} m \\
& =\sup _{\ell \in[t-\tau, t]} \int_{\ell+\mathcal{T}}^{t+\mathcal{T}}(-\epsilon(m)-\mu(m)) \mathrm{d} m \\
& =\sup _{\ell \in[t-\tau, t]} \int_{\ell}^{t}(-\epsilon(m+\mathcal{T})-\mu(m+\mathcal{T})) \mathrm{d} m .
\end{aligned}
$$

Since both $\mu$ and $\epsilon$ are periodic of period $\mathcal{T}$, it follows that

$$
\begin{aligned}
& \kappa(t+\mathcal{T})= \\
& \sup _{\ell \in[t-\tau, t]} \int_{\ell}^{t}(-\epsilon(m)-\mu(m)) \mathrm{d} m=\kappa(t) .
\end{aligned}
$$

From the fact that $\kappa, \epsilon$ and $\mu$ are all periodic of period $\mathcal{T}$, we easily deduce that for all $t \geq 0$, we have

$$
\begin{aligned}
& \sup _{t \geq 0}\left[\left(e^{\kappa(t)}-1\right) b(t)-\epsilon(t)\right] \leq-\varpi, \text { where } \\
& \varpi=-\sup _{t \in[0, \mathcal{T}]}\left[\left(e^{\kappa(t)}-1\right) b(t)-\epsilon(t)\right]
\end{aligned}
$$

The corollary now follows from Theorem 1 .

\subsection{First Illustration of Corollary 2}

Consider the one dimensional system

$$
\dot{x}(t)=-x(t)+b(t) x(t-\tau(t))
$$


where $b: \mathbb{R} \rightarrow \mathbb{R}$ is periodic of period $\mathcal{T}=1$ and such that there are constants $c \in(0,1)$ and $d>0$ satisfying (i) $b(t)=0$ for all $t \in[0, c)$, (ii) $b(t)=d$ for all $t \in[c, 1)$, and (iii) $d(1-c) \in(0,1)$. Assume that $\tau:[0, \infty) \rightarrow[0, \infty)$ is continuous and bounded by a constant $\bar{\tau}>0$. This is more general than the systems in Mazenc et al. (2015), since (95) has a time-varying delay. See Section 6.5 for a higher dimensional example.

Since $b$ is periodic, we apply Corollary 2. With $V(x)=$ $|x|$ and $a(t)=-1$, Assumption 1 is satisfied. Next, we give conditions ensuring that Assumption 9 holds. We first apply the method from Section 6.2. Let

$$
\epsilon_{*}=-\int_{0}^{1}[-1+b(m)] \mathrm{d} m=1-d(1-c) .
$$

Using the notation from Section 6.2 , we set $\bar{b}=d$ and $\mu(s)=a(s)+b(s)=-1+b(s)$, which gives

$$
\begin{aligned}
& s_{\mu}=\sup _{s \in[0,1]}\left(-\epsilon_{*}-\mu(s)\right)= \\
& \sup _{s \in[0,1]}(d(1-c)-b(s))=d(1-c) .
\end{aligned}
$$

Therefore, the delay bound $\tau_{*}$ from (81) in Section 6.2 is

$$
\tau_{*}=\frac{1}{s_{\mu}} \ln \left(1+\frac{\epsilon_{*}}{\bar{b}}\right)=\frac{1}{d(1-c)} \ln \left(\frac{1+c d}{d}\right) .
$$

To get less restrictive conditions than those obtained with $\epsilon(t)=\epsilon_{*}$, pick any constant $\nu \in\left(0, \min \left\{1, \epsilon_{*} / c\right\}\right)$, and let $\epsilon$ be the period 1 function such that (a) $\epsilon(t)=\nu$ for all $t \in[0, c)$ and (b) $\epsilon(t)=\frac{\epsilon_{*}-c \nu}{1-c}$ for all $t \in[c, 1)$. Then $\epsilon$ has positive upper and lower bounds. The function $\kappa$ in $(7)$ is

$$
\kappa(t)=\sup _{\ell \in[t-\bar{\tau}, t]} \int_{\ell}^{t}[-\epsilon(m)+1-b(m)] \mathrm{d} m .
$$

Also, our choice (96) of $\epsilon_{*}$ gives

$$
\int_{0}^{1}(\epsilon(m)-1+b(m)) \mathrm{d} m=0,
$$

so (76) from Assumption 9 is satisfied. Hence, all requirements from Assumption 9 will be satisfied if

$$
\sup _{t \in[0,1]}\left[\left(e^{\kappa(t)}-1\right) b(t)-\epsilon(t)\right]<0 \text {. }
$$

Since $b=0$ on $[0, c)$ and $\epsilon$ is positive valued, it follows that (100) is equivalent to

$$
\exp \left(\sup _{t \in[c, 1]} \kappa(t)\right)<\frac{1-c \nu}{d(1-c)} .
$$

Now observe that

$$
\begin{aligned}
& -\epsilon(m)+1-b(m)=-\left[\frac{1-c \nu}{1-c}-d\right]+1-d= \\
& c \frac{\nu-1}{1-c}<0
\end{aligned}
$$

for all $m \in[c, 1)$, while

$$
-\epsilon(m)+1-b(m)=-\nu+1
$$

for all $m \in[0, c)$. We deduce from (99) that

$$
\sup _{t \in[c, 1]} \kappa(t) \leq \bar{\tau}(1-\nu) \text {. }
$$

Then (101) is satisfied if

$$
\bar{\tau}<\frac{1}{1-\nu} \ln \left(\frac{1-c \nu}{d(1-c)}\right) .
$$

Taking the limit from the right as $\nu \rightarrow 0^{+}$in (103), we get $\bar{\tau}<\tau_{*}$, where

$$
\tau_{*}=-\ln (d(1-c)) .
$$

This is a less restrictive upper bound for $\tau$ than (98), since for instance, if we take $c=0.94$ and $d=10$, then $d(1-c)=$ $0.6 \in(0,1)$, and the difference between the delay bounds (98) and (104) is

$$
-\ln (d(1-c))-\frac{1}{d(1-c)} \ln \left(\frac{1+c d}{d}\right)>0.44 .
$$

This justifies our use of the more complicated function $\epsilon(t)$, instead of the constant $\epsilon_{*}$.

\subsection{Second Illustration of Corollary 2}

Consider the system

$$
\dot{x}=-m(t) m^{\top}(t) u(t-\tau(t))
$$

where $x$ is valued in $\mathbb{R}^{n}$ for any $n$, the function $m: \mathbb{R} \rightarrow \mathbb{R}^{n}$ is continuous and has some period $\mathcal{T}>0$, the input $u$ is valued in $\mathbb{R}^{n}$, and the delay $\tau$ is a time-varying piecewise continuous function that is bounded by a constant $\bar{\tau}>0$.

The system (106) arises in identification theory; see Mazenc et al. (2008). In Mazenc et al. (2008), we studied (106), but only in the special case where $\tau$ is constant. The approach in Mazenc et al. (2008) is based on the construction of a Lyapunov-Krasovskii functional, which is written as the sum of a strict Lyapunov function for the corresponding undelayed system, plus a double integral term in which the integrand is a function of the norm of the state. Here, we use Corollary 2 to provide stabilizability conditions in cases where the delay is time-varying. Notice that Mazenc et al. (2013) does not apply to systems with a time-varying delay. We first introduce:

\section{Assumption 10 The matrix $M \in \mathbb{R}^{n \times n}$ defined by}

$$
M=\frac{1}{\mathcal{T}} \int_{0}^{\mathcal{T}} m(s) m^{\top}(s) \mathrm{d} s
$$

is positive definite.

We define the constants

$$
k_{m}=\frac{1}{\mathcal{T}} \int_{0}^{\mathcal{T}}|m(s)|^{2} \mathrm{~d} s \text { and } s_{m}=\sup _{t \in[0, \mathcal{T}]}|m(t)|
$$

and let $\lambda_{M}$ be the smallest eigenvalue of $M$. Then $k_{m}>0$, $s_{m}>0$ and $\lambda_{M}>0$. We now add three assumptions:

Assumption 11 The inequality

$$
\frac{\lambda_{M}}{2+\mathcal{T} s_{m}^{2}}>\left(s_{m}^{4} \bar{\tau}^{2}+2 \mathcal{T} k_{m}\right) k_{m}
$$

is satisfied.

Assumption 12 The function $|m(t)|$ is globally Lipschitz, with some Lipschitz constant $l_{m}>0$. 
Assumption 13 The inequality

$$
\begin{aligned}
& 2\left(s_{m}^{4} \bar{\tau}^{2}+2 \mathcal{T} k_{m}\right) s_{m} l_{m} \bar{\tau} \mathcal{T}< \\
& \ln \left(1+\frac{\frac{2 \lambda_{M}}{2+\mathcal{T} s_{m}^{2}}-2\left(s_{m}^{4} \bar{\tau}^{2}+2 \mathcal{T} k_{m}\right) k_{m}}{\bar{b}}\right)
\end{aligned}
$$

is satisfied, where $\bar{b}=2\left(s_{m}^{4} \bar{\tau}^{2}+2 \mathcal{T} k_{m}\right) s_{m}^{2}$.

One can easily determine values of $\bar{\tau}$ such that (110) is satisfied.

Corollary 3 Let the system (106) satisfy Assumptions 1013. Then (106), in closed loop with the feedback $u(x(t))=$ $x(t)$, is globally uniformly exponentially stable to the origin.

Proof: The feedback produces the system

$$
\dot{x}=-m(t) m^{\top}(t) x(t-\tau(t))
$$

so we prove that the origin of (111) is globally uniformly exponentially stable. This system is a linear time-varying system and therefore we can restrict our stability analysis to the time interval $[2 \bar{\tau}, \infty)$.

For all $t \geq 2 \bar{\tau}$, we can rewrite the system as

$$
\begin{aligned}
\dot{x}= & -m(t) m^{\top}(t) x(t)-m(t) m^{\top}(t) \\
& \times \int_{t-\tau(t)}^{t} m(s) m^{\top}(s) x(s-\tau(s)) \mathrm{d} s .
\end{aligned}
$$

Let us consider $\nu_{1}(x)=\frac{1}{2}|x|^{2}$ and

$$
\nu_{2}(t, x)=\frac{1}{\mathcal{T}} x^{\top}\left(\int_{t-\mathcal{T}}^{t} \int_{\ell}^{t} m(s) m^{\top}(s) \mathrm{d} s \mathrm{~d} \ell\right) x
$$

By (112), we deduce that along all trajectories of (111), we have

$$
\begin{aligned}
& \dot{\nu}_{1}(t)=-\left(m^{\top}(t) x(t)\right)^{2}-x(t)^{\top} m(t) m^{\top}(t) \\
& \quad \times \int_{t-\tau(t)}^{t} m(s) m^{\top}(s) x(s-\tau(s)) \mathrm{d} s
\end{aligned}
$$

and

$$
\begin{aligned}
& \dot{\nu}_{2}(t)=\left(m^{\top}(t) x(t)\right)^{2}-x(t)^{\top} M x(t)- \\
& \frac{2}{\mathcal{T}} x(t)^{\top}\left(\int_{t-\mathcal{T}}^{t} \int_{\ell}^{t} m(s) m^{\top}(s) \mathrm{d} s \mathrm{~d} \ell\right) m(t) m^{\top}(t) x(t-\tau(t)),
\end{aligned}
$$

where $M$ is the matrix defined in (107).

Let us introduce the function $V(t, x)=2 \nu_{1}(x)+\nu_{2}(t, x)$. Then for all $t \geq 0$ and $x \in \mathbb{R}^{n}$, the inequalities

$$
\frac{1}{2}|x|^{2} \leq V(t, x) \leq \frac{1}{2}\left(2+\mathcal{T} s_{m}^{2}\right)|x|^{2}
$$

are satisfied. For all $t \geq 2 \bar{\tau}$, we get

$$
\begin{aligned}
& \dot{V}(t)= \\
& -x(t)^{\top} M x(t)-\left(m^{\top}(t) x(t)\right)^{2}-2\left\{x(t)^{\top} m(t)\right\} \\
& \times\left\{m^{\top}(t) \int_{t-\tau(t)}^{t} m(s) m^{\top}(s) x(s-\tau(s)) \mathrm{d} s\right\} \\
& -\frac{2}{\mathcal{T}} x(t)^{\top}\left(\int_{t-\mathcal{T}}^{t} \int_{\ell}^{t} m(s) m^{\top}(s) \mathrm{d} s \mathrm{~d} \ell\right) \\
& \times m(t) m^{\top}(t) x(t-\tau(t)) .
\end{aligned}
$$

Using Young's inequality to get $2 a b \leq a^{2}+b^{2}$ where $a$ and $b$ correspond to the terms in curly braces in (116), we obtain

$$
\begin{aligned}
& \dot{V}(t) \leq-x(t)^{\top} M x(t)+ \\
& \left|m^{\top}(t) \int_{t-\tau(t)}^{t} m(s) m^{\top}(s) x(s-\tau(s)) \mathrm{d} s\right|^{2} \\
& -\frac{2}{\mathcal{T}} x(t)^{\top}\left(\int_{t-\mathcal{T}}^{t} \int_{\ell}^{t} m(s) m^{\top}(s) \mathrm{d} s \mathrm{~d} \ell\right) \\
& \quad \times m(t) m^{\top}(t) x(t-\tau(t)) .
\end{aligned}
$$

Then Jensen's inequality gives

$$
\begin{aligned}
& \dot{V}(t) \leq-x(t)^{\top} M x(t)+\tau(t)|m(t)|^{2} \\
& \quad \times \int_{t-\tau(t)}^{t}\left|m(s) m^{\top}(s)\right|^{2}|x(s-\tau(s))|^{2} \mathrm{~d} s \\
& +\frac{2|m(t)|^{2}}{\mathcal{T}}\left(\int_{t-\mathcal{T}}^{t} \int_{\ell}^{t}|m(s)|^{2} \mathrm{~d} s \mathrm{~d} \ell\right)|x(t)||x(t-\tau(t))| .
\end{aligned}
$$

From the definition of $k_{m}$ in (108) and our choice of $\lambda_{M}$, it follows that

$$
\begin{aligned}
\dot{V}(t) \leq & -x(t)^{\top} M x(t)+\tau(t)|m(t)|^{2} \\
& \times \int_{t-\tau(t)}^{t}\left|m(s) m^{\top}(s)\right|^{2}|x(s-\tau(s))|^{2} \mathrm{~d} s \\
& +2|m(t)|^{2} \mathcal{T} k_{m}|x(t)||x(t-\tau(t))| \\
\leq & -\lambda_{M}|x(t)|^{2} \\
& +2 \mathcal{T} k_{m}|m(t)|^{2}|x(t)||x(t-\tau(t))| \\
& +\tau(t) s_{m}^{4}|m(t)|^{2} \int_{t-\tau(t)}^{t}|x(s-\tau(s))|^{2} \mathrm{~d} s .
\end{aligned}
$$

Hence, our bounds (115) give

$$
\begin{aligned}
& \dot{V}(t) \leq-\frac{2 \lambda_{M}}{2+\mathcal{T} s_{m}^{2}} V(t, x(t))+2 \tau(t) s_{m}^{4}|m(t)|^{2} \\
& \times \int_{t-\tau(t)}^{t} V(s-\tau(s), x(s-\tau(s))) \mathrm{d} s \\
& +4 \mathcal{T} k_{m}|m(t)|^{2} \sqrt{V(t, x(t)) V(t-\tau(t), x(t-\tau(t)))} .
\end{aligned}
$$

It follows that

$$
\begin{aligned}
\dot{V}(t) \leq & -\frac{2 \lambda_{M}}{2+\mathcal{T} s_{m}^{2}} V(t, x(t)) \\
& +2 \tau^{2}(t) s_{m}^{4}|m(t)|^{2} \sup _{s \in[t-2 \bar{\tau}, t]} V(s, x(s)) \\
& +4 \mathcal{T} k_{m}|m(t)|^{2} \sup _{s \in[t-2 \bar{\tau}, t]} V(s, x(s)) \\
\leq & -\frac{2 \lambda_{M}}{2+\mathcal{T} s_{m}^{2}} V(t, x(t)) \\
& +2\left(\bar{\tau}^{2} s_{m}^{4}+2 \mathcal{T} k_{m}\right)|m(t)|^{2} \sup _{s \in[t-2 \bar{\tau}, t]} V(s, x(s)) .
\end{aligned}
$$

We next apply Corollary 2 with

$$
a(t)=-\frac{2 \lambda_{M}}{2+\mathcal{T} s_{m}^{2}} \text { and } b(t)=2\left(s_{m}^{4} \bar{\tau}^{2}+2 \mathcal{T} k_{m}\right)|m(t)|^{2} .
$$

Then our choices $\mu=a+b$ and $\epsilon(t)=\epsilon_{*}$ from (78) in Section 6.2 and $k_{m}$ from (108) give

$$
\begin{aligned}
& \epsilon(t)=\epsilon_{*}=-\frac{1}{\mathcal{T}} \int_{0}^{\mathcal{T}} \mu(\ell) \mathrm{d} \ell=\frac{2 \lambda_{M}}{2+\mathcal{T} s_{m}^{2}} . \\
& -2\left(s_{m}^{4} \bar{\tau}^{2}+2 \mathcal{T} k_{m}\right) k_{m}
\end{aligned}
$$

From (109), it follows that $\epsilon_{*}>0$. Moreover, $\mu$ is globally 
Lipschitz with $l_{\mu}=4\left(s_{m}^{4} \bar{\tau}^{2}+2 \mathcal{T} k_{m}\right) s_{m} l_{m}$ as Lipschitz constant. We deduce from our discussion of Corollary 2 in Section 6.2 that

$$
l_{\mu} \bar{\tau} \mathcal{T}<2 \ln \left(1+\frac{\epsilon_{*}}{\sup _{t \in[0, \mathcal{T}]} b(t)}\right)
$$

ensures global asymptotic stability; see (89). It holds if (110) is satisfied. This concludes the proof.

\section{Other Examples}

\subsection{First Illustration of Corollary 1}

First consider the one dimensional system

$$
\dot{x}(t)=-[1+2 \cos (t)] x(t-\tau)
$$

with $\tau \geq 0$ being constant. Determining conditions ensuring that this system is exponentially stable is challenging because it is a time-varying system whose stability is caused by a term with delay and because no obvious Lyapunov-Krasovskii functional can be proposed.

For all $t \geq 2 \tau$, we have

$$
\begin{aligned}
& \dot{x}(t)= \\
& -[1+2 \cos (t)] x(t)+[1+2 \cos (t)] \int_{t-\tau}^{t} \dot{x}(m) \mathrm{d} m \\
& =-[1+2 \cos (t)] x(t) \\
& -\int_{t-2 \tau}^{t-\tau}[1+2 \cos (t)][1+2 \cos (m+\tau)] x(m) \mathrm{d} m .
\end{aligned}
$$

We analyze the system (123) via Corollary 1. Choosing $\mathcal{V}(x)=|x|$ and $\eta=2 \tau$, we get

$$
\begin{aligned}
& \dot{\mathcal{V}}(t) \leq-[1+2 \cos (t)] \mathcal{V}(x(t)) \\
& +\int_{t-\eta}^{t-\frac{1}{2} \eta}|1+2 \cos (t) \| 1+2 \cos (m+\tau)| \mathcal{V}(x(m)) \mathrm{d} m .
\end{aligned}
$$

We choose $\omega=0, a(t)=-[1+2 \cos (t)], c(m, t)=\mid 1+$ $2 \cos (t)|| 1+2 \cos (m+\tau) \mid$, and $b=0$. By (40), we have

$$
\begin{aligned}
h(t) \leq & \int_{t-\eta}^{t} e^{\int_{t}^{m+\eta}[1+2 \cos (\ell)] \mathrm{d} \ell} c(t, m+\eta) \mathrm{d} m \\
\leq & \left|1+2 \cos \left(t+\frac{\eta}{2}\right)\right| \\
& \times \int_{t-\eta}^{t} \mathcal{I}(m, \eta, t)|1+2 \cos (m+\eta)| \mathrm{d} m,
\end{aligned}
$$

where $\mathcal{I}(m, \eta, t)=e^{m+\eta-t+2(\sin (m+\eta)-\sin (t))}$. Since $|\sin (m+\eta)-\sin (t)| \leq|m+\eta-t|$, it follows from Hölder's inequality that

$$
\begin{aligned}
h(t) \leq & \left|1+2 \cos \left(t+\frac{\eta}{2}\right)\right| \\
& \times \int_{t-\eta}^{t} e^{m+\eta-t+2(m+\eta-t)}|1+2 \cos (m+\eta)| \mathrm{d} m \\
= & \left|1+2 \cos \left(t+\frac{\eta}{2}\right)\right| \\
& \times \int_{t}^{t+\eta} e^{3(m-t)}|1+2 \cos (m)| \mathrm{d} m \\
\leq & \left|1+2 \cos \left(t+\frac{\eta}{2}\right)\right| \\
& \times \sqrt{\frac{e^{6 \eta}-1}{6} \int_{t}^{t+\eta}(1+2 \cos (m))^{2} \mathrm{~d} m} \\
= & \frac{\sqrt{e^{6 \eta}-1}}{\sqrt{6}}\left|1+2 \cos \left(t+\frac{\eta}{2}\right)\right| \\
& \times \sqrt{\int_{t}^{t+\eta}\left(1+4 \cos (m)+4 \cos ^{2}(m)\right) \mathrm{d} m}
\end{aligned}
$$

Let us choose $T=\tau=2 \pi$. Then

$$
\begin{aligned}
& \int_{t}^{t+2 \pi}[a(\ell)+h(\ell)] \mathrm{d} \ell \\
& \leq-2 \pi+\frac{\sqrt{e^{6 \eta}-1}}{\sqrt{6}} \int_{t}^{t+2 \pi}\left|1+2 \cos \left(\ell+\frac{\eta}{2}\right)\right| \mathcal{G}_{a}(\eta, \ell) \mathrm{d} \ell \\
& \leq-2 \pi+\frac{\sqrt{e^{6 \eta}-1}}{\sqrt{6}} 3 \sqrt{\eta} \int_{t}^{t+2 \pi}\left|1+2 \cos \left(\ell+\frac{\eta}{2}\right)\right| \mathrm{d} \ell,
\end{aligned}
$$

where

$$
\begin{aligned}
& \mathcal{G}_{a}(\eta, \ell)=(3 \eta+4 \sin (\ell+\eta)- \\
& \left.4 \sin (\ell)+2 \int_{\ell}^{\ell+\eta} \cos (2 m) \mathrm{d} m\right)^{1 / 2} .
\end{aligned}
$$

Since cos is periodic of period $2 \pi$, we deduce that

$$
\begin{aligned}
& \int_{t}^{t+2 \pi}[a(\ell)+h(\ell)] \mathrm{d} \ell \\
& \leq-2 \pi+\frac{\sqrt{e^{6 \eta}-1}}{\sqrt{2}} \sqrt{3 \eta} \int_{0}^{2 \pi}|1+2 \cos (\ell)| \mathrm{d} \ell \\
& =-2 \pi+\sqrt{6 \eta\left(e^{6 \eta}-1\right)} \int_{0}^{\pi}|1+2 \cos (\ell)| \mathrm{d} \ell,
\end{aligned}
$$

where we also used the fact that

$$
\begin{aligned}
& \int_{\pi}^{2 \pi}|1+2 \cos (\ell)| \mathrm{d} \ell=\int_{\pi}^{2 \pi}|1+2 \cos (2 \pi-\ell)| \mathrm{d} \ell= \\
& \int_{0}^{\pi}|1+2 \cos (\ell)| \mathrm{d} \ell .
\end{aligned}
$$

Hence, Assumption 6 will be satisfied if the right side of (126) is negative, i.e., if

$$
\begin{aligned}
\sqrt{\eta\left(e^{6 \eta}-1\right)} & <\frac{\sqrt{2} \pi}{\sqrt{3} \int_{0}^{\pi}|1+2 \cos (\ell)| \mathrm{d} \ell} \\
& =\frac{\sqrt{2} \pi}{\sqrt{3}(\pi / 3+2 \sqrt{3})}
\end{aligned}
$$

holds, so this is a sufficient condition for the conclusions of Corollary 1 to hold.

\subsection{Second Illustration of Corollary 1}

We choose the system

$$
\dot{x}(t)=\sin (t) x(t)+u(t-\tau)
$$

and the control $u(t)=-[\sin (t+\tau)+1] x(t)$. Then the closed loop system can be rewritten as $\dot{x}(t)=-x(t)+[\sin (t)+$ $1][x(t)-x(t-\tau)]$ and so also as

$$
\begin{aligned}
& \dot{x}(t)=-x(t)+\int_{t-\tau}^{t}[\sin (t)+1] \sin (m) x(m) \mathrm{d} m \\
& -\int_{t-2 \tau}^{t-\tau}[\sin (t)+1][\sin (m+\tau)+1] x(m) \mathrm{d} m
\end{aligned}
$$

for all $t \geq 2 \tau$. Along all trajectories of (129), the function $V(x)=|x|$ satisfies

$$
\begin{aligned}
\dot{V}(t) \leq & -V(x(t))+\int_{t-\tau}^{t}[\sin (t)+1] V(x(m)) \mathrm{d} m \\
& +\int_{t-2 \tau}^{t-\tau}[\sin (t)+1][\sin (m+\tau)+1] V(x(m)) \mathrm{d} m \\
\leq & -V(x(t))+ \\
& \int_{t-2 \tau}^{t}[\sin (t)+1][|\sin (m+\tau)|+1] V(x(m)) \mathrm{d} m .
\end{aligned}
$$

We apply Corollary 1 with $b=0, T=2 \pi, \eta=2 \tau$, and $\omega=0$.

We set $a(t)=-1$ and

$$
c(m, t)=(\sin (t)+1)(|\sin (m+\tau)|+1) .
$$


To apply Corollary 1, we need the function

$$
\begin{aligned}
n(t)= & \frac{1}{T} \int_{t-T}^{t}\left[a(m)+e^{-\int_{m}^{m+\tau} a(\ell) \mathrm{d} \ell} b(m+\tau)\right. \\
& \left.+\int_{m-\eta}^{m} e^{-\int_{m}^{\ell+\eta} a(s) d s} c(m, \ell+\eta) \mathrm{d} \ell\right] \mathrm{d} m \\
= & \frac{1}{2 \pi} \int_{t-2 \pi}^{t}\left[-1+\int_{m-\eta}^{m} e^{\ell+\eta-m}[\sin (\ell+\eta)+1]\right. \\
& \left.\times\left[\left|\sin \left(m+\frac{\eta}{2}\right)\right|+1\right] \mathrm{d} \ell\right] \mathrm{d} m \\
= & -1+\frac{1}{2 \pi} \int_{t-2 \pi}^{t}[|\sin (m+\eta / 2)|+1] \\
& \times \int_{m-\eta}^{m} e^{\ell+\eta-m}[|\sin (\ell+\eta)|+1] \mathrm{d} \ell \mathrm{d} m
\end{aligned}
$$

to have a negative upper bound. We find sufficient conditions for such an upper bound to exist. To this end, first note that since

$$
(|\sin (m+\eta / 2)|+1) \int_{m-\eta}^{m} e^{\ell+\eta-m}[|\sin (\ell+\eta)|+1] \mathrm{d} \ell
$$

is a periodic function of $m$ having period $2 \pi$ in $m$, we get

$$
\begin{aligned}
n(t)= & -1+\frac{1}{2 \pi} \int_{0}^{2 \pi}(|\sin (m+\eta / 2)|+1) \\
& \times \int_{0}^{\eta} e^{\ell}[|\sin (\ell+m)|+1] \mathrm{d} \ell \mathrm{d} m .
\end{aligned}
$$

Next observe that

$$
\begin{aligned}
n(t) & \leq-1+\frac{e^{\eta}-1}{\pi} \int_{0}^{2 \pi}(|\sin (m+\eta / 2)|+1) \mathrm{d} m \\
& =-1+\frac{e^{\eta}-1}{\pi} 4 \pi=-5+4 e^{\eta} .
\end{aligned}
$$

Hence, the upper bound will exist provided $\eta<\ln (5 / 4)$. Hence, we get the stability condition

$$
\tau<\frac{1}{2} \ln \left(\frac{5}{4}\right)
$$

Then the proof of Corollary 1 provides a LyapunovKrasovskii functional construction.

\subsection{Discrete Time Example}

We illustrate Theorem 3. Let $p>1$ be an integer, and define $\left\{\beta_{1}, \beta_{2}, \ldots, \beta_{p}\right\}$ by

$$
\beta_{k}=\frac{1}{2(p+1)} \text { for all } k \in\{1, \ldots, p-1\} \text { and } \beta_{p}=3 \text {. }
$$

Then we extend $\left\{\beta_{1}, \beta_{2}, \ldots, \beta_{p}\right\}$ to form a sequence, by setting $\beta_{k+p}=\beta_{k}$ for all $k \in \mathbb{N}$. Then for each $k \geq 0$, the set $\left\{\beta_{1+k}, \beta_{2+k}, \ldots, \beta_{p+k}\right\}$ has exactly one 3 . For each $j \in\{0, \ldots, p\}$, we define the sequence $\left\{\alpha_{k, j}\right\}$ by $\alpha_{k, j}=\beta_{k}$ for all $k \in \mathbb{N}$. Then (69)-(70) become $\bar{\alpha}_{k}=(p+1) \beta_{k}$ and

$$
\alpha_{*}=\left(\prod_{i=1+j}^{p+j}(p+1) \beta_{i}\right)^{\frac{1}{p}}=\left(\frac{3}{2^{p-1}(p+1)^{p-2}}\right)^{\frac{1}{p}} .
$$

Consequently, $\alpha_{*}<1$ is equivalent to

$$
p>1+\frac{\ln (3(p+1))}{\ln (2(p+1))},
$$

which holds if $p>3$. This provides sufficient conditions for Assumption 7 to hold, with $a_{1}=1 / 2$ and $a_{2}=3(p+1)$. Also, Assumption 8 will be satisfied if

$$
\left(\frac{3}{2^{p-1}(p+1)^{p-2}}\right)^{1 / p}<\frac{1}{2^{p /(p+1)}}
$$

which can be satisfied if we choose $p$ large enough, and then Assumptions 7-8 will both be satisfied. Hence, we have given sufficient conditions for the assumptions of Theorem 3 to hold.

\section{Conclusions}

Time delay systems with time-varying delays play a central role in controls, but their stability analysis is beyond the scope of traditional Lyapunov-Krasovskii functional or other standard techniques. To help overcome these significant challenges, we applied a strictification approach to extend Razumikhin's theorem for time-varying systems. The approach entails converting a nonstrict Lyapunov-like function into a strict one, but was beyond the scope of the strictification methods in Malisoff and Mazenc (2009). A key feature of our analysis is that we do not require the Lyapunov functional to decay along trajectories when the delay is set to 0 , and our strictification analysis is outside the scope of existing results on the Razumikhin approach. We illustrated how our method compares with results that were obtained in earlier literature. We also provided new constructions for Lyapunov-Krasovskii functionals for time-varying delay systems, as well as analogous results for discrete time systems. In future work, we plan to extend our analysis to adaptive cases where the objectives include both tracking and parameter identification.

\section{Acknowledgement}

We thank Profs. Zhou and Egorov for sharing their inspiring information about their work on delay systems.

\section{References}

Astrom, K. and B. Wittenmark (1996). ComputerControlled Systems: Theory and Design, Third Edition. Dover Publications. New York.

Bekiaris-Liberis, N. and M. Krstic (2013a). Nonlinear Control under Nonconstant Delays. Society for Industrial and Applied Mathematics. Philadelphia, PA.

Bekiaris-Liberis, N. and M. Krstic (2013b). Robustness of nonlinear predictor feedback laws to timeand state-dependent delay perturbations. Automatica 49(6), 1576-1590.

Bresch-Pietri, D. and N. Petit (2014). Robust compensation of a chattering time-varying input delay. In Proceedings of the IEEE Conference on Decision and Control. Los Angeles, CA. pp. 457-462.

Cloosterman, M., N. van de Wouw, W. Heemels and H. Nijmeijer (2007). Stability of networked control systems with large delays. In Proceedings of the IEEE Conference on Decision and Control. New Orleans, LA. pp. 50175022 .

Dieulot, J.-Y. and J-P. Richard (2001). Tracking control of a nonlinear system with input-dependent delay. In Proceedings of the IEEE Conference on Decision and Control. Orlando, FL. pp. 4027-4031.

Downey, R., R. Kamalapurkar, N. Fischer and W. Dixon (2016). Compensating for fatigue-induced time-varying 
delayed muscle response in neuromuscular electrical stimulation control. Vol. 4 of Advances in Delays and Dynamics. Springer. New York. pp. 143-161.

Elaydi, S. and S. Zhang (1994). Stability and periodicity of difference equations with finite delay. Funkcialaj Ekvacioj 37(3), 401-413.

Fridman, E. and S-I. Niculescu (2008). On complete Lyapunov-Krasovskii functional techniques for uncertain systems with fast-varying delays. International Journal of Robust and Nonlinear Control 18(3), 364-374.

$\mathrm{Gu}, \mathrm{K}$. and S-I. Niculescu (2003). Survey on recent results in the stability and control of time-delay systems. Journal of Dynamic Systems, Measurement and Control 125(2), 158-165.

$\mathrm{Gu}, \mathrm{K} ., \mathrm{V}$. Kharitonov and J. Chen (2003). Stability of Time-Delay Systems. Birkhauser. Boston, MA.

Hale, J. and S. Verduyn-Lunel (1993). Introduction to Functional Differential Equations. Springer. New York.

Karafyllis, I. and M. Krstic (2012). Nonlinear stabilization under sampled and delayed measurements, and with inputs subject to delay and zero-order hold. IEEE Trans. Automat. Control 57(5), 1141-1154.

Malisoff, M. and F. Mazenc (2009). Constructions of Strict Lyapunov Functions. Springer-Verlag. London.

Marquez, M., I. Boussaada, H. Mounier and S-I. Niculescu (2015). Analysis and Control of Oilwell Drilling Vibrations: A Time-Delay Systems Approach. Springer. New York.

Mazenc, F. and M. Malisoff (2015a). Reduction model approach for systems with a time-varying delay. In Proceedings of the IEEE Conference on Decision and Control. Osaka, Japan. pp. 7723-7727.

Mazenc, F. and M. Malisoff (2015b). Trajectory based approach for the stability analysis of nonlinear systems with time delays. IEEE Trans. Automat. Control 60(6), 1716-1721.

Mazenc, F. and M. Malisoff (2016). Extension of Razumikhin's theorem for time-varying systems with delay. In Proceedings of the American Control Conference. Boston, MA. pp. 84-88.

Mazenc, F. and S-I. Niculescu (2011). Generating positive and stable solutions through delayed state feedback. $A u$ tomatica 47(3), 525-533.

Mazenc, F., M. Malisoff and S-I. Niculescu (2014). Reduction model approach for linear time-varying systems with delays. IEEE Trans. Automat. Control 59(8), 2068 2082.

Mazenc, F., M. Malisoff and S-I. Niculescu (2015). Stability analysis for systems with time-varying delay: trajectory based approach. In Proceedings of the IEEE Conference on Decision and Control. Osaka, Japan. pp. 1811-1816.

Mazenc, F., M. Malisoff and T. Dinh (2013). Robustness of nonlinear systems with respect to delay and sampling of the controls. Automatica 49(6), 1925-1931.

Mazenc, F., M. Malisoff and Z. Lin (2008). Further results on input-to-state stability for nonlinear systems with delayed feedbacks. Automatica 44(9), 2415-2421.

Mondié, S. and W. Michiels (2003). Finite spectrum assignment of unstable time-delay system with a safe imple- mentation. IEEE Trans. Automat. Control48(12), 22072212 .

Montagner, V., V. Leite, S. Tarbouriech and P. Peres (2005). Stability and stabilizability of discrete-time switched linear systems with state delay. In Proceedings of the American Control Conference. Portland, OR. pp. 3806-3811.

Pepe, P. and Z-P. Jiang (2006). A Lyapunov-Krasovskii methodology for ISS and iISS of time-delay systems. Systems and Control Letters 55(12), 1006-1014.

Pepe, P., I. Karafyllis and Z-P. Jiang (2008). On the Lyapunov-Krasovskii methodology for the ISS of systems described by coupled delay differential and difference equations. Automatica 44(9), 2266-2273.

Petit, N., Y. Creff and P. Rouchon (1998). Motion planning for two classes of nonlinear systems with delays depending on the control. In Proceedings of the IEEE Conference on Decision and Control. Tampa, FL. pp. 10071011.

Richard, J.-P. (2003). Time-delay systems: an overview of some recent advances and open problems. Automatica 39(10), 1667-1694.

Zhou, B. (2014). Truncated Predictor Feedback for TimeDelay Systems. Springer. London, UK. 